\title{
Time-Frequency Integrals and the Stationary Phase Method in Problems of Waves Propagation from Moving Sources ${ }^{\star}$
}

\author{
Gennadiy BURLAK ${ }^{\dagger}$ and Vladimir RABINOVICH ${ }^{\ddagger}$ \\ † Centro de Investigación en Ingeniería y Ciencias Aplicadas, Universidad Autónoma \\ del Estado de Morelos, Cuernavaca, Mor. México \\ E-mail: gburlak@uaem.mx \\ $\ddagger$ National Polytechnic Institute, ESIME Zacatenco, D.F. México \\ E-mail: vladimir.rabinovich@gmail.com
}

Received July 29, 2012, in final form December 02, 2012; Published online December 10, 2012 http://dx.doi.org/10.3842/SIGMA.2012.096

\begin{abstract}
The time-frequency integrals and the two-dimensional stationary phase method are applied to study the electromagnetic waves radiated by moving modulated sources in dispersive media. We show that such unified approach leads to explicit expressions for the field amplitudes and simple relations for the field eigenfrequencies and the retardation time that become the coupled variables. The main features of the technique are illustrated by examples of the moving source fields in the plasma and the Cherenkov radiation. It is emphasized that the deeper insight to the wave effects in dispersive case already requires the explicit formulation of the dispersive material model. As the advanced application we have considered the Doppler frequency shift in a complex single-resonant dispersive metamaterial (Lorenz) model where in some frequency ranges the negativity of the real part of the refraction index can be reached. We have demonstrated that in dispersive case the Doppler frequency shift acquires a nonlinear dependence on the modulating frequency of the radiated particle. The detailed frequency dependence of such a shift and spectral behavior of phase and group velocities (that have the opposite directions) are studied numerically.
\end{abstract}

Key words: dispersive media; two-dimensional stationary phase method; electromagnetic wave; moving modulated source

2010 Mathematics Subject Classification: 78A25; 78A35

\section{Introduction}

The paper is devoted to applications of time-frequency integrals and the two-dimensional stationary phase method for problems of waves propagation from moving sources in dispersive media. We consider the electromagnetic fields generated by a moving in a dispersive media modulated source of the form

$$
\boldsymbol{F}(t, \boldsymbol{x})=\boldsymbol{a}(t) e^{-i \omega_{0} t} \delta\left(\boldsymbol{x}-\boldsymbol{x}_{0}(t)\right), \quad t \in \mathbb{R}, \quad \boldsymbol{x}=\left(x_{1}, x_{2}, x_{3}\right) \in \mathbb{R}^{3},
$$

where $\omega_{0}$ is an eigenfrequency of the source, $\boldsymbol{a}(t)$ is a slowly varying amplitude, $\boldsymbol{x}_{0}(t)=$ $\left(x_{01}(t), x_{02}(t), x_{03}(t)\right)$ is a vector-function defining a motion of the source, $\delta$ is the standard $\delta$-function.

Some assumptions with respect to sources allow us to introduce a large dimensionless parameter $\lambda>0$ which characterizes simultaneously a slowness of variations of amplitudes and velocities

\footnotetext{
*This paper is a contribution to the Special Issue "Superintegrability, Exact Solvability, and Special Functions". The full collection is available at http://www.emis.de/journals/SIGMA/SESSF2012.html
} 
of sources, and large distances between sources and receivers. We obtain a representation of the fields as double oscillating integrals depending on the parameter $\lambda>0$

$$
\boldsymbol{\Phi}_{\lambda}(t, \boldsymbol{x})=\int_{\mathbb{R} \times \mathbb{R}} \boldsymbol{F}(t, \boldsymbol{x}, \omega, \tau, \lambda) e^{i \lambda S(t, \boldsymbol{x}, \omega, \tau)} \mathrm{d} \omega \mathrm{d} \tau,
$$

where $\boldsymbol{F}$ is a complex vector-valued amplitude and $S$ is a real-valued for $|\omega|$ large enough phase. Generally speaking integral (1.1) is divergent and we consider its regularization which is called the oscillatory integral.

Applying to the integrals (1.1) the stationary phase method we obtain the asymptotics of electromagnetic fields for large $\lambda>0$. We consider non uniformly moving sources in isotropic dispersive homogeneous media, in a particular case, in the isotropic plasma. Note that this method can be apply also for the analysis of waves propagation from moving sources in anisotropic dispersive non homogeneous media and media with negative phase velocity (metamaterials), and also for a motion with a velocity larger than a phase velocity of media (the Cherenkov radiation).

We would like to note that the asymptotic estimates of one-dimensional integrals are standard tools of the electrodynamics (see for instance [17, Chapters 3, 4], [33]) and go back to A. Sommerfeld [44], and L. Brillouin [8, Chapter 1]. But in the case of modulated non uniformly moving sources the representation of the fields in a form of a one-dimensional integral is impossible. In turn, a representation of fields as double time-frequency oscillating integrals with a subsequent asymptotic analysis yields effective formulae for both the fields and for the Doppler shifts. In particular, it gives new approaches to the Cherenkov radiation (see e.g. [2,3,7,19,21,25] and [29, Chapter 14]). In particular, the works [1,2,4-48,50-56] describe properties of the charged particle field in different dispersive media including traditional resonant medium [1, 2, 4-48, 51], active medium [51], anisotropic medium [5], left-handed medium [14,18,31], and so-called "wiremetamaterial" [53]. Some of these works develop a method of analysis of the moving charge field using complex function theory methods $[5,51]$. The papers $[13,50]$ are devoted to investigation of the fields of moving oscillators in different media.

The electromagnetic radiation from moving sources is a classical problem of the electrodynamics, and for the isotropic non dispersive media the solution of this problem is given by the Liènard-Wiechert potential (see for instance [28, Chapter VIII], [24, Chapter 14]). But the Liènard-Wiechert potential is not applicable for dispersive media and our representation is an effective tool for the investigation of electromagnetic fields generated by moving sources with a variable velocity.

Note that the above-described method for estimating of the acoustic field generated by moving sources in stratified acoustic waveguide has been proposed first in [23], and later on in the papers $[22,34,35]$.

There is another asymptotic approach to the problems of waves propagation from moving sources in dispersive media. It is the ray method in the space of the variables $(t, \boldsymbol{x})$ (see for instance $[4,30])$. Despite the fact that the ray method is applicable to a wider range of problems than the method suggested in the article, its implementation is encountering very serious difficulties in solving the ray and transport equations. By contrast the approach proposed here leads to simple, having a clear physical meaning, equations for the stationary points, and explicit formulae for electromagnetic fields and Doppler shifts. In particular, in the developed approach the distinction between the phase and group velocities appear in a natural way.

The paper is organized as follows. In Section 2 we give auxiliary material concerning the oscillatory integrals and the multidimensional stationary phase method. In Section 3 we consider electromagnetic waves propagation from moving modulated sources in dispersive medias. We obtain effective asymptotic formulae for the electromagnetic fields, Doppler effects, and retarded time. Section 4 devoted to applications obtained in Section 4 formulae. We consider a motion with a constant velocity in non dispersive media, electromagnetic field generated by modulated 
stationary sources in dispersive media, electromagnetic waves propagation from uniformly moving sources in the lossless no magnetized plasma (see e.g. $[19,24,47,48]$ ). We also formulate the equations for the Cherenkov radiation in dispersive medias in terms of representation (1.1) and the stationary phase method.

Further it is emphasized that the deeper comprehension and insight the wave effects in dispersive case already requires the explicit formulation of the dispersive material model. As the advanced application of the developed technique in Section 5 it is considered the Doppler frequency shift in a complex single-resonant dispersive metamaterial (Lorenz) model where in some frequency ranges the negativity of the real part of the refraction index can be reached $[36,39$, $42,43,52]$. We have demonstrated that in dispersive case the Doppler frequency shift acquires a nonlinear dependence on the modulating frequency of the radiated particle. (In dispersiveless medium such a function is linear.) The detailed frequency dependence of such shift and spectral behavior of phase and group velocities (that have the opposite directions) are studied numerically. Discussion and conclusions from our results are found in the last section.

\section{Auxiliary material: stationary phase method for the oscillatory integrals}

$1^{0}$. We use the standard notations for the spaces of differentiable functions: $C^{\infty}\left(\mathbb{R}^{n}\right)$ is the space of all infinitely differentiable functions on $\mathbb{R}^{n}, C_{b}^{\infty}\left(\mathbb{R}^{n}\right)$ is a subspace of $C^{\infty}\left(\mathbb{R}^{n}\right)$ consisting of functions bounded with all their partial derivatives, $C_{0}^{\infty}\left(\mathbb{R}^{n}\right)$ is a subspace of $C^{\infty}\left(\mathbb{R}^{n}\right)$ consisting of functions with a compact supports.

$2^{0}$. We consider integrals of the form

$$
\int_{\mathbb{R}^{n}} \boldsymbol{f}(\boldsymbol{x}) e^{i S(\boldsymbol{x})} \mathrm{d} \boldsymbol{x}
$$

where $\mathbb{R}^{n} \ni \boldsymbol{x} \rightarrow \boldsymbol{f}(\boldsymbol{x}) \in \mathbb{C}^{m}$ is called the amplitude and the scalar function $S$ is called the phase. We suppose that $\boldsymbol{f}$ and $S$ are infinitely differentiable (the existence only of a finite number of derivatives is necessary) and satisfy the following conditions: for every multiindex $\alpha$ there exists $C_{\alpha}>0$ such that

$$
\left|\partial^{\alpha} \boldsymbol{f}(\boldsymbol{x})\right| \leq C_{\alpha}\langle\boldsymbol{x}\rangle^{k}, \quad \boldsymbol{x} \in \mathbb{R}^{n}, \quad\langle\boldsymbol{x}\rangle=\left(1+|\boldsymbol{x}|^{2}\right)^{\frac{1}{2}},
$$

(i) $S(x)$ is a real function for $|x|$ is large enough,

(ii) for every $\alpha:|\alpha| \geq 2$ there exists $C_{\alpha}>0$ such that $\left|\partial^{\alpha} S(\boldsymbol{x})\right| \leq C_{\alpha}$,

(iii) there exists $C>0$ and $\rho>0$ such that

$$
|\nabla S(\boldsymbol{x})| \geq C|\boldsymbol{x}|^{\rho}
$$

for $|\boldsymbol{x}|$ large enough.

Note that if $k \geq-n$ integral (2.1) does not exist as absolutely convergent and we need a regularization of integral (2.1). Let $\chi \in C_{0}^{\infty}\left(\mathbb{R}^{n}\right)$, and $\chi(\boldsymbol{x})=1$ in a small neighborhood of the origin. We set $\chi_{R}(\boldsymbol{x})=\chi(\boldsymbol{x} / R)$.

Proposition 1 ( [41, Chapter 1]). Let estimate (2.2) and conditions (i)-(iii) hold. Then there exists a limit

$$
\boldsymbol{F}=\lim _{R \rightarrow \infty} \int_{\mathbb{R}^{n}} \chi_{R}(\boldsymbol{x}) \boldsymbol{f}(\boldsymbol{x}) e^{i S(\boldsymbol{x})} \mathrm{d} \boldsymbol{x}
$$

independent of the choose of the function $\chi$. 
Proof. We introduce the first-order differential operator $L$

$$
L u(\boldsymbol{x})=\left(1+|\nabla S(\boldsymbol{x})|^{2}\right)^{-1}(I-i \nabla S(\boldsymbol{x}) \cdot \nabla) u(\boldsymbol{x}), \quad \boldsymbol{x} \in \mathbb{R}^{n} .
$$

One can see that

$$
L e^{i S(\boldsymbol{x}, \boldsymbol{y})}=e^{i S(\boldsymbol{x}, \boldsymbol{y})} .
$$

Let $L^{\tau}$ be the transpose to $L$ differential operator. Then taking into the account (2.4) and applying the integration by parts we obtain

$$
\boldsymbol{F}_{R}=\int_{\mathbb{R}^{n}} \chi_{R}(\boldsymbol{x}) \boldsymbol{f}(\boldsymbol{x}) e^{i S(\boldsymbol{x})} \mathrm{d} \boldsymbol{x}=\int_{\mathbb{R}^{n}}\left(L^{\tau}\right)^{j}\left(\chi_{R}(\boldsymbol{x}) \boldsymbol{f}(\boldsymbol{x})\right) e^{i S(\boldsymbol{x})} \mathrm{d} \boldsymbol{x} .
$$

Conditions $(i)-($ iii $)$ yield

$$
\left|\left(L^{\tau}\right)^{j}\left(\chi_{R}(\boldsymbol{x}) \boldsymbol{f}(\boldsymbol{x})\right)\right| \leq C_{j}\langle\boldsymbol{x}\rangle^{k-\rho j},
$$

with the constant $C_{j}>0$ independent of $R>0$. Let $j>\frac{k+n}{\rho}$, then the integral in the right side part of (2.5) is absolutely convergent, uniformly with respect to $R>0$, and we can go to the limit for $R \rightarrow \infty$ in (2.5). Hence the limit in (2.3) exists, independent of $\chi$, and

$$
\boldsymbol{F}=\lim _{R \rightarrow \infty} \boldsymbol{F}_{R}=\int_{\mathbb{R}^{n}}\left(\left(L^{\tau}\right)^{j} \boldsymbol{f}(\boldsymbol{x})\right) e^{i S(\boldsymbol{x})} \mathrm{d} \boldsymbol{x},
$$

where $j>\frac{k+n}{\rho}$. The integrals defined by formula (2.6) are called oscillatory.

$3^{0}$. We consider an integral depending on a parameter $\lambda>0$

$$
\boldsymbol{I}_{\lambda}=\int_{\mathbb{R}^{n}} \boldsymbol{f}(\boldsymbol{x}) e^{i \lambda S(\boldsymbol{x})} \mathrm{d} \boldsymbol{x},
$$

where $\boldsymbol{f}, S$ satisfy condition $(2.2),(i)-(i i i)$. We say that $\boldsymbol{x}_{0}$ is a non-degenerate stationary point of the phase $S$ if

$$
\nabla S\left(\boldsymbol{x}_{0}\right)=0
$$

and

$$
\operatorname{det} S^{\prime \prime}\left(\boldsymbol{x}_{0}\right) \neq 0
$$

where $S^{\prime \prime}(\boldsymbol{x})=\left(\frac{\partial^{2} S(\boldsymbol{x})}{\partial x_{i} \partial x_{j}}\right)_{i, j=1}^{n}$ is the Hess matrix of the phase $S$ at the point $\boldsymbol{x}$.

Proposition 2 (see for instance $[6,16]$ ). Let there exist a finite set $\left\{\boldsymbol{x}_{1}, \ldots, \boldsymbol{x}_{N}\right\}$ of nondegenerate stationary points of the phase $S$. Then

$$
\boldsymbol{I}_{\lambda}=\sum_{j=1}^{N} \boldsymbol{F}_{j}(\lambda)
$$

where

$$
\boldsymbol{F}_{j}(\lambda)=\left(\frac{2 \pi}{\lambda}\right)^{\frac{n}{2}} \frac{\exp \left(i \lambda S\left(\boldsymbol{x}_{j}\right)+\frac{i \pi}{4} \operatorname{sgn} S^{\prime \prime}\left(\boldsymbol{x}_{j}\right)\right)}{\left|\operatorname{det} S^{\prime \prime}\left(\boldsymbol{x}_{j}\right)\right|^{1 / 2}} \boldsymbol{f}\left(\boldsymbol{x}_{j}\right)\left(1+O\left(\frac{1}{\lambda}\right)\right),
$$

and $\operatorname{sgn} S^{\prime \prime}\left(\boldsymbol{x}_{j}\right)$ is the difference between the number of positive and negative eigenvalues of the matrix $S^{\prime \prime}\left(\boldsymbol{x}_{j}\right)$. 


\section{Electromagnetic wave propagation in dispersive media}

The Maxwell equations in dispersive media are obtained by the replacing of the electric and magnetic permittivity $\varepsilon, \mu$ by the operators $\varepsilon\left(D_{t}\right), \mu\left(D_{t}\right)$ where

$$
\varepsilon\left(D_{t}\right) \boldsymbol{u}(t)=\frac{1}{2 \pi} \int_{-\infty}^{\infty} \varepsilon(\omega) \hat{\boldsymbol{u}}(\omega) e^{i \omega t} \mathrm{~d} \omega, \quad \mu\left(D_{t}\right) \boldsymbol{u}(t)=\frac{1}{2 \pi} \int_{-\infty}^{\infty} \mu(\omega) \hat{\boldsymbol{u}}(\omega) e^{i \omega t} \mathrm{~d} \omega,
$$

where the Fourier transform

$$
\hat{\boldsymbol{u}}(\omega)=\int_{-\infty}^{\infty} \boldsymbol{u}(t) e^{-i \omega t} \mathrm{~d} t
$$

is understood in the sense of distributions. Let

$$
c(\omega)=\frac{1}{\sqrt{\varepsilon(\omega) \mu(\omega)}}
$$

be a phase velocity, $k(\omega)=\frac{\omega}{c(\omega)}$ be a wave number.

We suppose (see [29, Chapter IX]) that:

(i) the functions $\varepsilon(\omega), \mu(\omega)$ are limits in the sense of the distributions of analytic bounded in the upper complex half-plane functions;

(ii) $k^{2}(\omega)$ has a finite number $\omega_{1}<\cdots<\omega_{k}$ of simple zeros on $\mathbb{R}$, and $\inf _{\omega \in \mathbb{R} \backslash\left[\omega_{1}-\epsilon, \omega_{k}+\epsilon\right]} k^{2}(\omega)>0$ for small enough $\epsilon>0$;

(iii) the group velocity $v_{g}(\omega)=\frac{1}{k^{\prime}(\omega)}>0$ for all $\omega \in \mathbb{R} \backslash\left[\omega_{1}-\epsilon, \omega_{k}+\epsilon\right]$.

The system of the Maxwell equations for dispersive medias is

$$
\begin{aligned}
& \boldsymbol{\nabla} \times \boldsymbol{H}=\varepsilon\left(D_{t}\right) \frac{\partial \boldsymbol{E}}{\partial t}+\boldsymbol{j}, \\
& \boldsymbol{\nabla} \times \boldsymbol{E}=\mu\left(D_{t}\right) \frac{\partial \boldsymbol{H}}{\partial t}, \\
& \varepsilon\left(D_{t}\right) \boldsymbol{\nabla} \cdot \boldsymbol{E}=\rho, \\
& \boldsymbol{\nabla} \cdot \boldsymbol{H}=0,
\end{aligned}
$$

with the continuity equation

$$
\nabla \cdot \boldsymbol{j}+\frac{\partial \rho}{\partial t}=0
$$

After the Fourier transform with respect to the time we obtain

$$
\begin{aligned}
& \nabla \times \hat{\boldsymbol{H}}(\omega)=i \varepsilon(\omega) \omega \hat{\boldsymbol{E}}(\omega)+\hat{\boldsymbol{j}}(\omega), \\
& \nabla \times \hat{\boldsymbol{E}}(\omega)=i \mu(\omega) \omega \hat{\boldsymbol{H}}(\omega), \\
& \varepsilon(\omega) \boldsymbol{\nabla} \cdot \hat{\boldsymbol{E}}(\omega)=\hat{\rho}(\omega), \\
& \nabla \cdot \hat{\boldsymbol{H}}(\omega)=0, \\
& \nabla \cdot \hat{\boldsymbol{j}}(\omega)-i \omega \hat{\rho}(\omega)=0 .
\end{aligned}
$$

In the standard way system (3.1), (3.2) is reduced to the pair of independent equations

$$
\begin{aligned}
& \nabla \times \nabla \times \hat{\boldsymbol{E}}(\omega)-k^{2}(\omega) \hat{\boldsymbol{E}}(\omega)=i \omega \mu(\omega) \hat{\boldsymbol{j}}(\omega), \\
& \nabla \times \boldsymbol{\nabla} \times \hat{\boldsymbol{H}}(\omega)-k^{2}(\omega) \hat{\boldsymbol{H}}(\omega)=\nabla \times \hat{\boldsymbol{j}}(\omega) .
\end{aligned}
$$


Unique solutions of equations (3.3), (3.4) in domain where $k^{2}(\omega)>0$ is defined by the limiting absorption principle. That is

$$
\hat{\boldsymbol{E}}(\omega)=\lim _{\epsilon \rightarrow+0} \hat{\boldsymbol{E}}_{\epsilon}(\omega), \quad \hat{\boldsymbol{H}}(\omega)=\lim _{\epsilon \rightarrow+0} \hat{\boldsymbol{H}}_{\epsilon}(\omega),
$$

where $\hat{\boldsymbol{E}}_{\epsilon}(\omega), \hat{\boldsymbol{H}}_{\epsilon}(\omega)$ are the unique bounded solutions of the equations

$$
\begin{aligned}
& \nabla \times \nabla \times \hat{\boldsymbol{E}}_{\epsilon}(\omega)-\left(k^{2}(\omega)+i \epsilon\right) \hat{\boldsymbol{E}}_{\epsilon}(\omega)=i \omega \mu(\omega) \hat{\boldsymbol{j}}(\omega), \\
& \boldsymbol{\nabla} \times \boldsymbol{\nabla} \times \hat{\boldsymbol{H}}_{\epsilon}(\omega)-\left(k^{2}(\omega)+i \epsilon\right) \hat{\boldsymbol{H}}_{\epsilon}(\omega)=\boldsymbol{\nabla} \times \hat{\boldsymbol{j}}(\omega),
\end{aligned}
$$

and $k(\omega)=i \sqrt{\left|k^{2}(\omega)\right|}$ is a purely imaginary number in domains where $k^{2}(\omega)<0$. For these $\omega$ equations (3.3), (3.4) have unique decreasing solutions. By using the relations

$$
\nabla \times \nabla \times \hat{\boldsymbol{E}}(\omega)=-\nabla^{2} \hat{\boldsymbol{E}}(\omega)+\nabla(\nabla \cdot \hat{\boldsymbol{E}}(\omega))
$$

and

$$
\boldsymbol{\nabla} \cdot \hat{\boldsymbol{E}}(\omega)=\varepsilon^{-1}(\omega) \hat{\rho}(\omega)
$$

we reduce (3.3) to the equation

$$
\begin{aligned}
\nabla^{2} \hat{\boldsymbol{E}}(\omega)+k^{2}(\omega) \hat{\boldsymbol{E}}(\omega) & =\varepsilon^{-1}(\omega) \boldsymbol{\nabla} \hat{\rho}(\omega)-i \omega \mu(\omega) \hat{\boldsymbol{j}}(\omega) \\
& =-i \omega \mu(\omega)\left(\hat{\boldsymbol{j}}(\omega)+\frac{1}{k^{2}(\omega)} \boldsymbol{\nabla}(\boldsymbol{\nabla} \cdot \hat{\boldsymbol{j}})(\omega)\right)=\boldsymbol{F}_{\omega}
\end{aligned}
$$

The similar way we obtain from equation (3.4) that

$$
\nabla^{2} \hat{\boldsymbol{H}}(\omega)+k^{2}(\omega) \hat{\boldsymbol{H}}(\omega)=-\nabla \times \hat{\boldsymbol{j}}(\omega)=\boldsymbol{\Phi}_{\omega} .
$$

Equations (3.5) and (3.6) are independent and can be used for the definition of $\hat{\boldsymbol{E}}$ and $\hat{\boldsymbol{H}}$.

Let $g_{\omega}(\boldsymbol{x})=\frac{e^{i k(\omega)|\boldsymbol{x}|}}{4 \pi|\boldsymbol{x}|}$ be the fundamental solution of the scalar Helmholtz equation

$$
\Delta g_{\omega}(\boldsymbol{x})+k^{2}(\omega) g_{\omega}(\boldsymbol{x})=-\delta(\boldsymbol{x}), \quad \boldsymbol{x} \in \mathbb{R}^{3},
$$

satisfying the limiting absorption principle. Hence the solutions of equations (3.5), (3.6) are given as

$$
\boldsymbol{E}(t, x)=\frac{1}{2 \pi} \int_{-\infty}^{\infty} e^{i \omega t}\left(g_{\omega} * \boldsymbol{F}_{\omega}\right)(\boldsymbol{x}) \mathrm{d} \omega, \quad \boldsymbol{H}(t, \boldsymbol{x})=\frac{1}{2 \pi} \int_{-\infty}^{\infty} e^{i \omega t}\left(g_{\omega} * \boldsymbol{\Phi}_{\omega}\right)(\boldsymbol{x}) \mathrm{d} \omega .
$$

where the convolution is understood in the sense of the distributions

$$
(g * \boldsymbol{\Psi})(\boldsymbol{x})=\int_{\mathbb{R}^{3}} \frac{e^{i k(\omega)|\boldsymbol{x}-\boldsymbol{y}|}}{4 \pi|\boldsymbol{x}-\boldsymbol{y}|} \boldsymbol{\Psi}(y) \mathrm{d} \boldsymbol{y} .
$$

Let

$$
\boldsymbol{j}(t, \boldsymbol{x})=A(t) \boldsymbol{v}(t) \delta\left(\boldsymbol{x}-\boldsymbol{x}_{0}(t)\right),
$$

where $\delta$ is the standard $\delta$-function, $\boldsymbol{v}(t)=\dot{\boldsymbol{x}}_{0}(t)$ is a velocity of a source, $A(t)$ is an amplitude of the source. Then (3.7) implies that

$$
\boldsymbol{H}(t, \boldsymbol{x})=\frac{1}{8 \pi^{2}} \int_{\mathbb{R}^{2}} \nabla_{\boldsymbol{x}} \times\left(\frac{e^{i\left(k(\omega)\left|\boldsymbol{x}-\boldsymbol{x}_{0}(\tau)\right|-\omega(t-\tau)\right)}}{\left|\boldsymbol{x}-\boldsymbol{x}_{0}(\tau)\right|} \boldsymbol{v}(\tau)\right) A(\tau) \mathrm{d} \omega \mathrm{d} \tau,
$$


and

$$
\boldsymbol{E}(t, \boldsymbol{x})=\frac{1}{8 \pi^{2} i} \int_{\mathbb{R}^{2}} A(\tau) \omega \mu(\omega)\left(I+k^{-2}(\omega) \nabla_{\boldsymbol{x}} \nabla_{\boldsymbol{x}} \cdot\right) \frac{e^{i\left(k(\omega)\left|\boldsymbol{x}-\boldsymbol{x}_{0}(\tau)\right|-\omega(t-\tau)\right)}}{\left|\boldsymbol{x}-\boldsymbol{x}_{0}(\tau)\right|} \boldsymbol{v}(\tau) \mathrm{d} \omega \mathrm{d} \tau
$$

Applying the analyticity of the integrand with respect to $\omega$ in the upper half-plane $\mathbb{C}_{+}$we deform the line of integration $(-\infty, \infty)$ with respect to $\omega$ into the contour $\Gamma=\left(-\infty, \omega_{1}-\varepsilon\right) \cup$ $\Gamma^{\prime} \cup\left(\omega_{k}+\varepsilon,+\infty\right)$, where $\Gamma^{\prime}$ is located in the upper complex half-plane and bypasses from above all singularities $\left\{\omega_{1}, \ldots, \omega_{k}\right\}$ of the integrand on the real line.

The phase of the double integrals is

$$
S(\omega, \tau)=k(\omega)\left|\boldsymbol{x}-\boldsymbol{x}_{0}(\tau)\right|+\omega \tau,
$$

and

$$
\frac{\partial S(\omega, \tau)}{\partial \omega}=\frac{\left|\boldsymbol{x}-\boldsymbol{x}_{0}(\tau)\right|}{v_{g}(\omega)}+\tau, \quad \frac{\partial S(\omega, \tau)}{\partial \tau}=-k(\omega) v(\tau, \boldsymbol{x})+\omega,
$$

where

$$
v(\tau, \boldsymbol{x})=\frac{\dot{\boldsymbol{x}}_{0}(\tau) \cdot\left(\boldsymbol{x}-\boldsymbol{x}_{0}(\tau)\right)}{\left|\boldsymbol{x}-\boldsymbol{x}_{0}(\tau)\right|}
$$

is the projection of the speed $\dot{\boldsymbol{x}}_{0}(\tau)$ on the vector $\boldsymbol{x}-\boldsymbol{x}_{0}(\tau)$. We suppose that there exists large enough $R>0$ such that

$$
\inf _{\omega^{2}+\tau^{2} \geq R^{2}}\left|\frac{v(\tau, \boldsymbol{x})}{v_{g}(\omega)}-1\right|>0, \quad \inf _{\omega^{2}+\tau^{2} \geq R^{2}}\left|\frac{v(\tau, \boldsymbol{x})}{c(\omega)}-1\right|>0 .
$$

Then the phase $S$ in integrals (3.9), (3.10) satisfies the estimate

$$
|\nabla S(\omega, \tau)| \geq C(|\omega|+|\tau|)
$$

in the domain $\left\{(\omega, \tau) \in \mathbb{R}^{2}: \omega^{2}+\tau^{2} \geq R^{2}\right\}$ where $C=C(R)>0$ for $R>0$ large enough. Hence integrals (3.9), (3.10) exist as oscillatory.

\subsection{Asymptotic analysis of the fields of moving sources}

Now we introduce a dimensionless parameter $\lambda>0$ as

$$
\lambda=\left(\inf _{t \in \mathbb{R}}\left|\boldsymbol{x}-\boldsymbol{x}_{0}(t)\right|\right) \frac{\Omega}{c_{0}}>0,
$$

where $c_{0}$ is the light speed in the vacuum, $\Omega>0$ is a characteristic frequency of the problem. In what follows we suppose that $\lambda$ is a large parameter, that frequently is a ratio of distance between the moving source and the receiver to the field wavelength. Such a distance is much more then $\left(\frac{\Omega}{c_{0}}\right)^{-1}$ for all the time. Since $a^{\prime}(t)=\frac{1}{\lambda} \tilde{a}^{\prime}(t / \lambda)=O\left(\frac{1}{\lambda}\right)$ (see equation (3.12)) the $1 / \lambda$ in general characterizes the slowness variations of the amplitude $a$ due to slowness variations of different parameters of a problem, e.g. charge trajectory, material dispersion, etc.

In what follows we will suppose that

$$
A(t)=a(t) e^{-i \omega_{0} t}
$$

where

$$
a(t)=\tilde{a}(t / \lambda),
$$


$\tilde{a} \in C_{b}^{\infty}(\mathbb{R}), \omega_{0}>0$ is an eigenfrequency (a carrier frequency) of the source,

$$
\boldsymbol{x}_{0}(t)=\lambda \boldsymbol{X}_{0}(t / \lambda), \quad t \in \mathbb{R}
$$

$\lambda>0$ is a large dimensionless parameter characterizing the slowness of variations of the amplitude $a$, and the velocity

$$
\dot{\boldsymbol{x}}_{0}(t)=\dot{\boldsymbol{X}}_{0}(t / \lambda), \quad t \in \mathbb{R}
$$

where the vector-function $\dot{\boldsymbol{X}}_{0}(t) \in C_{b}^{\infty}(\mathbb{R}) \otimes \mathbb{C}^{3}$.

To reduce integrals (3.9), (3.10) to a form containing the large parameter $\lambda>0$ we use the scale change of variables

$$
\boldsymbol{x}=\lambda \boldsymbol{X}, \quad t=\lambda T, \quad \tau=\lambda \iota .
$$

and obtain following representations for magnetic and electric fields

$$
\begin{aligned}
& \overline{\boldsymbol{H}}_{\lambda}(T, \boldsymbol{X})=\frac{1}{8 \pi^{2} \lambda} \int_{\Gamma \times \mathbb{R}} \tilde{a}(\iota) \nabla_{\boldsymbol{X}} \times\left(\frac{e^{i \lambda \bar{S}(T, \boldsymbol{X}, \omega, \iota)}}{\left|\boldsymbol{X}-\boldsymbol{X}_{0}(\iota)\right|} \boldsymbol{V}(\iota)\right) \mathrm{d} \omega \mathrm{d} \iota, \\
& \overline{\boldsymbol{E}}_{\lambda}(t, \boldsymbol{x})=\frac{1}{8 \pi^{2} i} \int_{\Gamma \times \mathbb{R}} \tilde{a}(\iota) \omega \mu(\omega)\left(I+\frac{1}{\lambda^{2} k^{2}(\omega)} \nabla_{\boldsymbol{x}} \nabla_{\boldsymbol{x}} \cdot\right) \frac{e^{i \lambda \bar{S}(T, \boldsymbol{X}, \omega, \iota)}}{\left|\boldsymbol{X}-\boldsymbol{X}_{0}(\iota)\right|} \boldsymbol{V}(\iota) \mathrm{d} \omega \mathrm{d} \iota .
\end{aligned}
$$

The phase $\bar{S}$ in integrals (3.14), (3.15) at $\lambda \rightarrow+\infty$ is

$$
\bar{S}(T, \boldsymbol{X}, \omega, \iota)=k(\omega)\left|\boldsymbol{X}-\boldsymbol{X}_{0}(\iota)\right|-\omega(T-\iota)-\omega_{0} \iota .
$$

Note that contributions in the main term of asymptotics of integrals (3.14), (3.15) are given by the stationary points of the phase $\bar{S}(T, \boldsymbol{X}, \omega, \iota)$ located in the domain $\mathbb{R} \backslash\left[\omega_{1}-\epsilon, \omega_{k}+\epsilon\right]$. The stationary points of $\bar{S}(T, \boldsymbol{X}, \omega, \iota)$ with respect to $(\omega, \iota)$ for fixed $(T, \boldsymbol{X})$ are solutions of the system

$$
\begin{aligned}
& \frac{\partial \bar{S}(T, \boldsymbol{X}, \omega, \iota)}{\partial \omega}=\frac{\left|\boldsymbol{X}-\boldsymbol{X}_{0}(\iota)\right|}{v_{g}(\omega)}-(T-\iota)=0, \\
& \frac{\partial \bar{S}(T, \boldsymbol{X}, \omega, \iota)}{\partial \iota}=-k(\omega) V(\boldsymbol{X}, \tau)+\left(\omega-\omega_{0}\right)=0,
\end{aligned}
$$

where

$$
V(\boldsymbol{X}, \tau)=\frac{\boldsymbol{X}-\boldsymbol{X}_{0}(\iota)}{\left|\boldsymbol{X}-\boldsymbol{X}_{0}(\iota)\right|} \cdot \boldsymbol{V}(\iota)
$$

is the value of the projection of $\boldsymbol{V}(\iota)$ on the vector $\boldsymbol{X}-\boldsymbol{X}_{0}(\iota)$.

Let $\omega_{s}=\omega_{s}(T, \boldsymbol{X}), \iota_{s}=\iota_{s}(T, \boldsymbol{X})$ be a non-degenerate stationary point of the phase $\bar{S}$. It means that $\left(\omega_{s}, \iota_{s}\right)$ is a solution of system (3.16) and

$$
\operatorname{det} \bar{S}^{\prime \prime}\left(T, \boldsymbol{X}, \omega_{s}, \iota_{s}\right) \neq 0,
$$

where

$$
\bar{S}^{\prime \prime}(T, \boldsymbol{X}, \omega, \iota)=\left(\begin{array}{cc}
k^{\prime \prime}(\omega)\left|\boldsymbol{X}-\boldsymbol{X}_{0}(\iota)\right| & 1-\frac{V(\boldsymbol{X}, \tau)}{v_{g}(\omega)} \\
1-\frac{V(\boldsymbol{X}, \tau)}{v_{g}(\omega)} & -k(\omega) \frac{\partial V(\boldsymbol{X}, \iota)}{\partial \iota}
\end{array}\right)
$$


is the Hess matrix of the phase $\bar{S}$. We denote by $\operatorname{sgn} \bar{S}^{\prime \prime}\left(T, \boldsymbol{X}, \omega_{s}, \iota_{s}\right)$ the difference between the number of positive and negative eigenvalues of the matrix $\bar{S}^{\prime \prime}\left(T, \boldsymbol{X}, \omega_{s}, \iota_{s}\right)$.

The contribution of the stationary point $\left(\omega_{s}, \iota_{s}\right)$ in the asymptotics of $\overline{\boldsymbol{H}}_{\lambda}(T, \boldsymbol{X}), \overline{\boldsymbol{E}}_{\lambda}(T, \boldsymbol{X})$ is given by the formulae (see Proposition 2)

$$
\begin{aligned}
\overline{\boldsymbol{H}}_{\lambda, s}(T, \boldsymbol{X})= & \frac{1}{4 \pi \lambda^{2}} \nabla_{\boldsymbol{X}} \times\left(\frac{e^{i \lambda \bar{S}\left(T, \boldsymbol{X}, \omega_{s}, \iota_{s}\right)}}{\left|\boldsymbol{X}-\boldsymbol{X}_{0}\left(\iota_{s}\right)\right|} \boldsymbol{V}\left(\iota_{s}\right)\right) \frac{e^{\frac{i \pi}{4} \operatorname{sgn} \bar{S}^{\prime \prime}\left(T, \boldsymbol{X}, \omega_{s}, \iota_{s}\right)}}{\left|\operatorname{det} \bar{S}^{\prime \prime}\left(T, \boldsymbol{X}, \omega_{s}, \iota_{s}\right)\right|^{1 / 2}} \tilde{a}\left(\iota_{s}\right) \\
& \times\left(1+O\left(\frac{1}{\lambda}\right)\right)
\end{aligned}
$$

and

$$
\begin{aligned}
\overline{\boldsymbol{E}}_{\lambda, s}(t, \boldsymbol{x})= & \frac{\tilde{a}\left(\iota_{s}\right)}{4 \pi \lambda i}\left[\omega_{s} \mu\left(\omega_{s}\right) I+\frac{1}{\lambda^{2} k^{2}\left(\omega_{s}\right)} \nabla_{\boldsymbol{x}} \nabla_{\boldsymbol{x}} \cdot \frac{e^{i \lambda \bar{S}\left(T, \boldsymbol{X}, \omega_{s}, \iota_{s}\right)}}{\left|\boldsymbol{X}-\boldsymbol{X}_{0}\left(\iota_{s}\right)\right|} \boldsymbol{V}\left(\iota_{s}\right)\right] \\
& \times \frac{e^{\frac{i \pi}{4} \operatorname{sgn} \bar{S}^{\prime \prime}\left(T, \boldsymbol{X}, \omega_{s}, \iota_{s}\right)}}{\left|\operatorname{det} \bar{S}^{\prime \prime}\left(T, \boldsymbol{X}, \omega_{s}, \iota_{s}\right)\right|^{1 / 2}}\left(1+O\left(\frac{1}{\lambda}\right)\right) .
\end{aligned}
$$

If the phase $\bar{S}$ has a finite set of stationary points the main term of the asymptotics of the electromagnetic field is the sum of contributions of the every stationary point.

The expressions (3.17) and (3.18) can be simplified if we are restricted by the terms of the order $O\left(\frac{1}{\lambda}\right)$

$$
\begin{aligned}
& \overline{\boldsymbol{H}}_{\lambda, s}(T, \boldsymbol{X}) \\
& \sim \frac{i k\left(\omega_{s}\right) \tilde{a}\left(\iota_{s}\right)}{4 \pi \lambda} \frac{e^{i \lambda \bar{S}\left(T, \boldsymbol{X}, \omega_{s}, \iota_{s}\right)+\frac{i \pi}{4} \operatorname{sgn} \bar{S}^{\prime \prime}\left(T, \boldsymbol{X}, \omega_{s}, \iota_{s}\right)}\left(\nabla_{\boldsymbol{X}} \times \boldsymbol{V}\left(\iota_{s}\right)\right)\left|\boldsymbol{X}-\boldsymbol{X}_{0}\left(\iota_{s}\right)\right|}{\left|\operatorname{det} \bar{S}^{\prime \prime}\left(T, \boldsymbol{X}, \omega_{s}, \iota_{s}\right)\right|^{1 / 2}\left|\boldsymbol{X}-\boldsymbol{X}_{0}\left(\iota_{s}\right)\right|}
\end{aligned}
$$

and

$$
\begin{aligned}
\overline{\boldsymbol{E}}_{\lambda, s}(t, \boldsymbol{x}) \sim & \frac{\tilde{a}\left(\iota_{s}\right)}{4 \pi \lambda i}\left[\omega_{s} \mu\left(\omega_{s}\right) \boldsymbol{V}\left(\iota_{s}\right)-\left(\nabla_{\boldsymbol{x}} \nabla_{\boldsymbol{x}} \cdot \boldsymbol{V}\left(\iota_{s}\right)\right)\left|\boldsymbol{X}-\boldsymbol{X}_{0}\left(\iota_{s}\right)\right|\right] \\
& \times \frac{e^{i \lambda \bar{S}\left(T, \boldsymbol{X}, \omega_{s}, \iota_{s}\right)+\frac{i \pi}{4} \operatorname{sgn} \bar{S}^{\prime \prime}\left(T, \boldsymbol{X}, \omega_{s}, \iota_{s}\right)}}{\left|\boldsymbol{X}-\boldsymbol{X}_{0}\left(\iota_{s}\right)\right|\left|\operatorname{det} \bar{S}^{\prime \prime}\left(T, \boldsymbol{X}, \omega_{s}, \iota_{s}\right)\right|^{1 / 2}} .
\end{aligned}
$$

Coming back to the variables $(t, \boldsymbol{x})$ we obtain the following asymptotic formulae

$$
\begin{aligned}
\boldsymbol{H}_{s}(t, \boldsymbol{x}) \sim & \frac{1}{4 \pi} \nabla_{\boldsymbol{x}} \times\left(\frac{e^{i S\left(t, \boldsymbol{x}, \omega_{s}, \tau_{s}\right)}}{\left|\boldsymbol{x}-\boldsymbol{x}_{0}\left(\tau_{s}\right)\right|} \boldsymbol{v}\left(\tau_{s}\right)\right) \frac{a\left(\tau_{s}\right) e^{\frac{i \pi}{4} \operatorname{sgn} S^{\prime \prime}\left(t, \boldsymbol{x}, \omega_{s}, \tau_{s}\right)}}{\left|\operatorname{det} S^{\prime \prime}\left(t, \boldsymbol{x}, \omega_{s}, \tau_{s}\right)\right|^{1 / 2}}, \\
\boldsymbol{E}_{s}(t, \boldsymbol{x}) \sim & \frac{1}{4 \pi i} a\left(\tau_{s}\right) \omega_{s} \mu\left(\omega_{s}\right)\left(I+\frac{1}{k^{2}\left(\omega_{s}\right)} \nabla_{\boldsymbol{x}} \nabla_{\boldsymbol{x}} \cdot\right) \frac{e^{i S\left(t, \boldsymbol{x}, \omega_{s}, \tau_{s}\right)}}{\left|\boldsymbol{x}-\boldsymbol{x}_{0}\left(\tau_{s}\right)\right|} \boldsymbol{v}\left(\tau_{s}\right) \\
& \times \frac{e^{\frac{i \pi}{4} \operatorname{sgn} S^{\prime \prime}\left(t, \boldsymbol{x}, \omega_{s}, \tau_{s}\right)}}{\left|\operatorname{det} S^{\prime \prime}\left(t, \boldsymbol{x}, \omega_{s}, \tau_{s}\right)\right|^{1 / 2}},
\end{aligned}
$$

where

$$
t=\frac{T}{\lambda}, \quad\left|\boldsymbol{x}-\boldsymbol{x}_{0}(t)\right|=\frac{\left|\boldsymbol{X}-\boldsymbol{X}_{0}(T)\right|}{\lambda}, \quad \lambda \rightarrow \infty .
$$

In formulae (3.21), (3.22) the phase is $S(t, \boldsymbol{x}, \omega, \tau)=k(\omega)\left|\boldsymbol{x}-\boldsymbol{x}_{0}(\tau)\right|-\omega(t-\tau)-\omega_{0} \tau$, and the stationary points $\left(\omega_{s}=\omega_{s}(t, \boldsymbol{x}), \tau_{s}=\tau_{s}(t, \boldsymbol{x})\right)$ are solutions of the system

$$
\frac{\partial S(t, \boldsymbol{x}, \omega, \tau)}{\partial \omega}=\frac{\left|\boldsymbol{x}-\boldsymbol{x}_{0}(\tau)\right|}{v_{g}(\omega)}-(t-\tau)=0,
$$




$$
\frac{\partial S(t, \boldsymbol{x}, \omega, \tau)}{\partial \tau}=-k(\omega) v(\boldsymbol{x}, \tau)+\left(\omega-\omega_{0}\right)=0,
$$

and

$$
S^{\prime \prime}(\boldsymbol{x}, t, \omega, \tau)=\left(\begin{array}{cc}
k^{\prime \prime}(\omega)\left|\boldsymbol{x}-\boldsymbol{x}_{0}(\tau)\right| & 1-\frac{v(\boldsymbol{x}, \tau)}{v_{g}(\omega)} \\
1-\frac{v(\boldsymbol{x}, \tau)}{v_{g}(\omega)} & -k(\omega) \frac{\partial v(\boldsymbol{x}, \tau)}{\partial \tau}
\end{array}\right) .
$$

Note that under conditions

$$
\begin{aligned}
& \sup _{(\omega, \tau) \in \mathbb{R}^{2}}\left(\frac{|\boldsymbol{v}(\tau)|}{\left|v_{g}(\omega)\right|}+\frac{\Omega}{T_{0}}\left|k^{\prime \prime}(\omega)\right|\left|\boldsymbol{x}-\boldsymbol{x}_{0}(\tau)\right|\right)<1, \\
& \sup _{(\omega, \tau) \in \mathbb{R}^{2}}\left(\frac{T_{0}}{\omega^{0}} k(\omega)\left|\frac{\partial v(\boldsymbol{x}, \tau)}{\partial \tau}\right|+\frac{|\boldsymbol{v}(\tau)|}{\left|v_{g}(\omega)\right|}\right)<1,
\end{aligned}
$$

where $\left(T_{0}, \Omega\right)$ are the scale time and frequency, system (3.23) has an unique solution which can be obtained by the method of successive approximations.

Coming to the variables $(t, \boldsymbol{x})$ in formulae (3.19), (3.20) we simplify formulae (3.21), (3.22)

$$
\begin{aligned}
\boldsymbol{H}_{s}(t, \boldsymbol{x}) \sim & \frac{i k\left(\omega_{s}\right) a\left(\tau_{s}\right)}{4 \pi} \frac{e^{i \lambda S\left(t, \boldsymbol{x}, \omega_{s}, \tau_{s}\right)+\frac{i \pi}{4} \operatorname{sgn} S^{\prime \prime}\left(t, \boldsymbol{x}, \omega_{s}, \tau_{s}\right)}\left(\nabla_{\boldsymbol{x}} \times \boldsymbol{v}\left(\tau_{s}\right)\right)\left(\left|\boldsymbol{x}-\boldsymbol{x}_{0}\left(\tau_{s}\right)\right|\right)}{\left|\operatorname{det} S^{\prime \prime}\left(t, \boldsymbol{x}, \omega_{s}, \iota_{s}\right)\right|^{1 / 2}\left|\boldsymbol{x}-\boldsymbol{x}_{0}\left(\tau_{s}\right)\right|}, \\
\boldsymbol{E}_{s}(t, \boldsymbol{x}) \sim & \frac{a\left(\iota_{s}\right)}{4 \pi i}\left[\omega_{s} \mu\left(\omega_{s}\right) \boldsymbol{v}\left(\tau_{s}\right)-\left(\nabla_{\boldsymbol{x}} \nabla_{\boldsymbol{x}} \cdot \boldsymbol{v}\left(\tau_{s}\right)\right)\left(\left|\boldsymbol{x}-\boldsymbol{x}_{0}\left(\tau_{s}\right)\right|\right)\right] \\
& \times \frac{e^{i \lambda S\left(t, \boldsymbol{x}, \omega_{s}, \tau_{s}\right)+\frac{i \pi}{4} \operatorname{sgn} S^{\prime \prime}\left(t, \boldsymbol{x}, \omega_{s}, \tau_{s}\right)}}{\left|\boldsymbol{x}-\boldsymbol{x}_{0}\left(\tau_{s}\right)\right|\left|\operatorname{det} S^{\prime \prime}\left(t, \boldsymbol{x}, \omega_{s}, \tau_{s}\right)\right|^{1 / 2}} .
\end{aligned}
$$

Example 1. Let $\boldsymbol{x}_{0}(\tau)=(0, v \tau, H)$. Then $\boldsymbol{x}-\boldsymbol{x}_{0}(\tau)=\left(x_{1}, x_{2}-v \tau, x_{3}-H\right)$,

$$
\left|\boldsymbol{x}-\boldsymbol{x}_{0}(\tau)\right|=\left(x_{1}^{2}+\left(x_{2}-v \tau\right)^{2}+\left(x_{3}-H\right)^{2}\right)^{1 / 2},
$$

$\boldsymbol{v}=(0, v, 0)$. The system for the stationary phase point $\left(\omega_{s}(t, \boldsymbol{x}), \tau_{s}(t, \boldsymbol{x})\right)$ is

$$
\begin{aligned}
& \frac{\left(x_{1}^{2}+\left(x_{2}-v \tau\right)^{2}+\left(x_{3}-H\right)^{2}\right)^{1 / 2}}{v_{g}(\omega)}-(t-\tau)=0, \\
& -k(\omega) \frac{v\left(x_{2}-v \tau\right)}{\left(x_{1}^{2}+\left(x_{2}-v \tau\right)^{2}+x_{3}^{2}\right)^{1 / 2}}+\left(\omega-\omega_{0}\right)=0 .
\end{aligned}
$$

For applying formulae (3.24), (3.25) we have to use

$$
\begin{aligned}
\left(\nabla_{\boldsymbol{x}} \times \boldsymbol{v}\right)\left|\boldsymbol{x}-\boldsymbol{x}_{0}(\tau)\right| & =\left(-v \frac{\partial\left|\boldsymbol{x}-\boldsymbol{x}_{0}(\tau)\right|}{\partial x_{3}}, 0, v \frac{\partial\left|\boldsymbol{x}-\boldsymbol{x}_{0}(\tau)\right|}{\partial x_{1}}\right) \\
& =\left(-v \frac{x_{3}-H}{\left|\boldsymbol{x}-\boldsymbol{x}_{0}(\tau)\right|}, 0, v \frac{x_{1}}{\left|\boldsymbol{x}-\boldsymbol{x}_{0}(\tau)\right|}\right),
\end{aligned}
$$

and

$$
\left(\nabla_{\boldsymbol{x}} \nabla_{\boldsymbol{x}} \cdot \boldsymbol{v}\right)\left(\left|\boldsymbol{x}-\boldsymbol{x}_{0}(\tau)\right|\right)=v\left(\frac{x_{1}\left(x_{2}-v \tau\right)}{\left|\boldsymbol{x}-\boldsymbol{x}_{0}(\tau)\right|^{2}}, \frac{x_{1}^{2}+\left(x_{3}-H\right)^{2}}{\left|\boldsymbol{x}-\boldsymbol{x}_{0}(\tau)\right|^{3}}, \frac{\left(x_{3}-H\right)\left(x_{2}-v \tau\right)}{\left|\boldsymbol{x}-\boldsymbol{x}_{0}(\tau)\right|^{2}}\right) .
$$




\subsection{Doppler effect and retarded time}

Note that for fix point $\boldsymbol{x}$ formulae (3.21), (3.22) can be written of the form

$$
\boldsymbol{W}(t)=\boldsymbol{A}(t) e^{i F(t)},
$$

where $\boldsymbol{A}(t)$ is a bounded vector-function, $F$ is a real-valued function such that $\lim _{t \rightarrow \infty} F(t)=\infty$. According to the signal processing theory (see for instance [12]) $F(t)$ is a phase of the wave process $\boldsymbol{W}(t)$, and the instantaneous frequency $\omega_{\text {in }}(t)$ of the wave process $\boldsymbol{W}(t)$ is defined as $\omega_{\text {in }}(t)=-F^{\prime}(t)$. In our case

$$
\begin{aligned}
F(t) & =S\left(t, \boldsymbol{x}, \omega_{s}(t, \boldsymbol{x}), \tau_{s}(t, \boldsymbol{x})\right) \\
& =k\left(\omega_{s}(t, \boldsymbol{x})\right)\left|\boldsymbol{x}-\boldsymbol{x}_{0}\left(\tau_{s}(t, \boldsymbol{x})\right)\right|-\omega_{s}(t, \boldsymbol{x})\left(t-\tau_{s}(t, \boldsymbol{x})\right)-\omega_{0} \tau_{s}(t, \boldsymbol{x}),
\end{aligned}
$$

where $\left(\omega_{s}(t, \boldsymbol{x}), \tau_{s}(t, \boldsymbol{x})\right)$ is a stationary point of the phase $S$. Differentiating of $F$ as a composed function we obtain

$$
\begin{aligned}
-F^{\prime}(t)= & -\frac{\partial S\left(t, \boldsymbol{x}, \omega_{s}(t, \boldsymbol{x}), \tau_{s}(t, \boldsymbol{x})\right)}{\partial t}-\frac{\partial S\left(t, \boldsymbol{x}, \omega_{s}(t, \boldsymbol{x}), \tau_{s}(t, \boldsymbol{x})\right)}{\partial \omega} \frac{\partial \omega_{s}(t, \boldsymbol{x})}{\partial t} \\
& -\frac{\partial S\left(t, \boldsymbol{x}, \omega_{s}(t, \boldsymbol{x}), \tau_{s}(t, \boldsymbol{x})\right)}{\partial \tau} \frac{\partial \tau_{s}(t, \boldsymbol{x})}{\partial t} .
\end{aligned}
$$

Taking into account that $\left(\omega_{s}(t, \boldsymbol{x}), \tau_{s}(t, \boldsymbol{x})\right)$ is the stationary point of $S$, we obtain that

$$
\omega_{\text {in }}(t)=\omega_{s}(t, \boldsymbol{x}) .
$$

It implies that the instantaneous frequency $\omega_{\text {in }}(t)$ of the wave processes $\boldsymbol{H}(t, \boldsymbol{x}), \boldsymbol{E}(t, \boldsymbol{x})$ for fixed $\boldsymbol{x}$ coincides with $\omega_{s}(t, \boldsymbol{x})$. Hence the instantaneous Doppler effect is

$$
\omega_{s}(t, \boldsymbol{x})-\omega_{0}=k\left(\omega_{s}(t, \boldsymbol{x})\right) v\left(\boldsymbol{x}, \tau_{s}(t, \boldsymbol{x})\right) .
$$

Considering the case $k\left(\omega_{s}(t, \boldsymbol{x})\right)>0$ we obtain the usual Doppler effect

$$
v\left(\boldsymbol{x}, \tau_{s}(t, \boldsymbol{x})\right)>0 \Longrightarrow \omega_{s}(t, \boldsymbol{x})>\omega_{0} \quad \text { and } \quad v\left(\boldsymbol{x}, \tau_{s}(t, \boldsymbol{x})\right)<0 \Longrightarrow \omega_{s}(t, \boldsymbol{x})<\omega_{0} .
$$

In the case $k\left(\omega_{s}(t, \boldsymbol{x})\right)<0$ (metamaterials) we obtain the inverse Doppler effect

$$
v\left(\boldsymbol{x}, \tau_{s}(t, \boldsymbol{x})\right)<0 \Longrightarrow \omega_{s}(t, \boldsymbol{x})<\omega_{0}, \quad \text { and } \quad v\left(\boldsymbol{x}, \tau_{s}(t, \boldsymbol{x})\right)>0 \Longrightarrow \omega_{s}(t, \boldsymbol{x})>\omega_{0} .
$$

It follows from formulae $(3.24),(3.25)$ that $\tau_{s}(t, \boldsymbol{x})$ is the excitation time of the signal arriving to the receiver located at the point $\boldsymbol{x}$ at the time $t$. Hence the mode Doppler effect for the time (the retarded time) is

$$
t-\tau_{s}(t, \boldsymbol{x})=\frac{\left|\boldsymbol{x}-\boldsymbol{x}_{0}\left(\tau_{s}(t, \boldsymbol{x})\right)\right|}{v_{g}\left(\omega_{s}(t, \boldsymbol{x})\right)}>0
$$

because the group velocity $v_{g}(\omega)>0$.

\section{Applications}

\subsection{Moving source in non dispersive medias}

We suppose here that the electric and magnetic permittivity $\varepsilon, \mu$, and hence the light speed $c=\frac{1}{\sqrt{\varepsilon \mu}}$ are independent of $\omega$. We consider a moving source of the form (3.8) where $A(t)$ and $\boldsymbol{x}_{0}(t)$ have form (3.11), (3.13). In this case

$$
S(t, \boldsymbol{x}, \omega, \tau)=\frac{\omega\left|\boldsymbol{x}-\boldsymbol{x}_{0}(\tau)\right|}{c}-\omega(t-\tau)-\omega_{0} \tau .
$$


System (3.23) accepts the form

$$
\frac{\left|\boldsymbol{x}-\boldsymbol{x}_{0}(\tau)\right|}{c}-(t-\tau)=0, \quad-\frac{\omega}{c} v(\boldsymbol{x}, \tau)+\left(\omega-\omega_{0}\right)=0
$$

and the first equation (4.1) independent of $\omega$. Note that under subliminal velocity of the source

$$
\sup _{t}|\boldsymbol{v}(t)|<c
$$

first equation in (4.1) has an unique solution $\tau_{s}=\tau_{s}(t, \boldsymbol{x})$ for every points $t, \boldsymbol{x}$. Second equation in (4.1) implies that

$$
\omega_{s}=\omega_{s}(t, \boldsymbol{x})=\frac{\omega_{0}}{1-\frac{v\left(\boldsymbol{x}, \tau_{s}\right)}{c}} .
$$

Moreover

$$
\operatorname{det} S^{\prime \prime}\left(t, \boldsymbol{x}, \omega_{s}, \tau_{s}\right)=-\left(1-\frac{v\left(\boldsymbol{x}, \tau_{s}\right)}{c}\right)^{2}
$$

and

$$
\operatorname{sgn} S^{\prime \prime}\left(t, \boldsymbol{x}, \omega_{s}, \tau_{s}\right)=0 .
$$

Substituting $\omega_{s}, \tau_{s}$, det $S^{\prime \prime}\left(\omega_{s}, \tau_{s}\right)$, sgn $S^{\prime \prime}\left(\omega_{s}, \tau_{s}\right)$ from (4.2), (4.3) in formulae (3.21), (3.22) we obtain the expression for $\boldsymbol{H}(t, \boldsymbol{x})$ and $\boldsymbol{E}(t, \boldsymbol{x})$

$$
\boldsymbol{H}(t, \boldsymbol{x}) \sim \frac{\nabla_{\boldsymbol{x}} \times\left(\frac{e^{i S\left(t, \boldsymbol{x}, \omega_{s}, \tau_{s}\right)}}{\left|\boldsymbol{x}-\boldsymbol{x}_{0}\left(\tau_{s}\right)\right|} \boldsymbol{v}\left(\tau_{s}\right)\right) a\left(\tau_{s}\right)}{4 \pi\left(1-\frac{v\left(\boldsymbol{x}, \tau_{s}\right)}{c}\right)},
$$

and

$$
\boldsymbol{E}(t, \boldsymbol{x}) \sim \frac{1}{4 \pi i} \frac{a\left(\tau_{s}\right) \omega_{s} \mu\left(\omega_{s}\right)}{\left(1-\frac{v\left(\boldsymbol{x}, \tau_{s}\right)}{c}\right)}\left(I+\frac{1}{k^{2}\left(\omega_{s}\right)} \nabla_{\boldsymbol{x}} \nabla_{\boldsymbol{x}} \cdot\right) \frac{e^{i S\left(t, \boldsymbol{x}, \omega_{s}, \tau_{s}\right)}}{\left|\boldsymbol{x}-\boldsymbol{x}_{0}\left(\tau_{s}\right)\right|} \boldsymbol{v}\left(\tau_{s}\right)
$$

for $t=\frac{T}{\lambda},\left|\boldsymbol{x}-\boldsymbol{x}_{0}(t)\right|=\frac{\left|\boldsymbol{X}-\boldsymbol{X}_{0}(T)\right|}{\lambda}, \lambda \rightarrow \infty$.

It should be noted that these formulae are asymptotic simplifications of the Liènard-Wiechert potentials [24, Chapter 14].

\subsection{Modulated stationary source in dispersive media}

Let us consider the electromagnetic field generated by a modulated stationary source of the form

$$
\boldsymbol{j}(t, \boldsymbol{x})=A(t) e^{-i \omega_{0} t} \delta\left(\boldsymbol{x}-\boldsymbol{x}_{0}\right) \boldsymbol{e}, \quad \omega_{0}>0, \quad A(t)=a(t / \lambda),
$$

where $\lambda>0$ is a large parameter, $\boldsymbol{e} \in \mathbb{R}^{3}$ is a unit vector, $\omega_{0}>0$ is an eigenfrequency of the source.

Repeating the calculations carried out for obtaining formulae (3.9), (3.10) we obtain

$$
\begin{aligned}
& \boldsymbol{H}(t, \boldsymbol{x})=\frac{1}{8 \pi^{2}} \int_{\mathbb{R}^{2}} \nabla_{\boldsymbol{x}} \times\left(\frac{e^{i\left(k(\omega)\left|\boldsymbol{x}-\boldsymbol{x}_{0}\right|-\omega(t-\tau)\right)}}{\left|\boldsymbol{x}-\boldsymbol{x}_{0}\right|} \boldsymbol{e}\right) A(\tau) \mathrm{d} \omega \mathrm{d} \tau \\
& \boldsymbol{E}(t, \boldsymbol{x})=\frac{1}{8 \pi^{2} i} \int_{\mathbb{R}^{2}} A(\tau) \omega \mu(\omega)\left(I+k^{-2}(\omega) \nabla_{\boldsymbol{x}} \nabla_{\boldsymbol{x}} \cdot\right)\left(\frac{e^{i\left(k(\omega)\left|\boldsymbol{x}-\boldsymbol{x}_{0}\right|-\omega(t-\tau)\right)}}{\left|\boldsymbol{x}-\boldsymbol{x}_{0}\right|} \boldsymbol{e}\right) \mathrm{d} \omega \mathrm{d} \tau .
\end{aligned}
$$


The further asymptotic analyses of $\boldsymbol{H}(t, \boldsymbol{x}), \boldsymbol{E}(t, \boldsymbol{x})$ is completely similar to given in Section 4.1. The phase $S$ in this case is

$$
S(t, \boldsymbol{x}, \omega, \tau)=k(\omega)\left|\boldsymbol{x}-\boldsymbol{x}_{0}\right|-\omega(t-\tau)-\omega_{0} \tau .
$$

Hence system (3.23) accepts the form

$$
\tau=t-\frac{\left|\boldsymbol{x}-\boldsymbol{x}_{0}\right|}{v_{g}(\omega)}, \quad \omega=\omega_{0},
$$

The phase $S(t, \boldsymbol{x}, \omega, \tau)$ has the unique stationary point

$$
\omega_{s}=\omega_{0}, \quad \tau_{s}=t-\frac{\left|\boldsymbol{x}-\boldsymbol{x}_{0}\right|}{v_{g}\left(\omega_{0}\right)} \quad \text { and } \quad S^{\prime \prime}\left(t, \boldsymbol{x}, \omega_{0}, \tau_{s}\right)=\left(\begin{array}{cc}
k^{\prime \prime}\left(\omega_{0}\right)\left|\boldsymbol{x}-\boldsymbol{x}_{0}\right| & 1 \\
1 & 0
\end{array}\right) .
$$

Hence $\operatorname{det} S^{\prime \prime}\left(t, \boldsymbol{x}, \omega_{0}, \tau_{s}\right)=-1, \operatorname{sgn} S\left(t, \boldsymbol{x}, \omega_{0}, \tau_{s}\right)=0$ and

$$
S\left(t, \boldsymbol{x}, \omega_{0}, \tau_{s}\right)=k\left(\omega_{0}\right)\left|\boldsymbol{x}-\boldsymbol{x}_{0}\right|-\omega_{0} \tau_{s} .
$$

It implies that

$$
\begin{aligned}
& \boldsymbol{H}(t, \boldsymbol{x}) \sim \frac{1}{4 \pi} a\left(t-\frac{\left|\boldsymbol{x}-\boldsymbol{x}_{0}\right|}{v_{g}\left(\omega_{0}\right)}\right) \nabla_{\boldsymbol{x}} \times\left(\frac{e^{i\left(k\left(\omega_{0}\right)\left|\boldsymbol{x}-\boldsymbol{x}_{0}\right|-\omega_{0} \tau_{s}\right)}}{\left|\boldsymbol{x}-\boldsymbol{x}_{0}\right|} \boldsymbol{e}\right), \\
& \boldsymbol{E}(t, \boldsymbol{x}) \sim \frac{\omega_{s} \mu\left(\omega_{s}\right) a\left(t-\frac{\left|\boldsymbol{x}-\boldsymbol{x}_{0}\right|}{v_{g}\left(\omega_{0}\right)}\right)}{4 \pi i}\left(I+\frac{1}{k^{2}\left(\omega_{0}\right)} \nabla_{\boldsymbol{x}} \nabla_{\boldsymbol{x}} \cdot\right) \frac{e^{i S\left(t, \boldsymbol{x}, \omega_{0}, \tau_{s}\right)}}{\left|\boldsymbol{x}-\boldsymbol{x}_{0}\left(\tau_{s}\right)\right|} \boldsymbol{e}
\end{aligned}
$$

for the "large" time and distance between the source and the receiver. Note that the retarded time is $t-\frac{\left|\boldsymbol{x}-\boldsymbol{x}_{0}\right|}{v_{g}\left(\omega_{0}\right)}$.

\subsection{Propagation from a moving source in the plasma}

We consider a lossless no magnetized plasma whose the collision frequency equals to zero (see for instance $[19,47,48])$. Hence the constitutive parameters in plasma are

$$
\varepsilon(\omega)=\varepsilon_{0}\left(1-\frac{\omega_{p}^{2}}{\omega^{2}}\right), \quad \mu=\mu_{0},
$$

$\varepsilon_{0}, \mu_{0}$ are the electric and magnetic permittivity of the vacuum,

$$
\omega_{p}^{2}=\frac{n q^{2}}{m \varepsilon_{0}}
$$

where $\omega_{p}$ is the plasma frequency, $n$ is the particle density, $m, q$ are the mass and charge of the electron. Hence the phase velocity in the plasma is

$$
c(\omega)=\frac{c_{0}}{\sqrt{1-\frac{\omega_{p}^{2}}{\omega^{2}}}},
$$

the wave-number is

$$
k(\omega)=\frac{\sqrt{\omega^{2}-\omega_{p}^{2}}}{c_{0}},
$$


and the group velocity is

$$
v_{g}(\omega)=c_{0} \sqrt{1-\frac{\omega_{p}^{2}}{\omega^{2}}}
$$

where $c_{0}$ is the light speed in the vacuum.

We consider the electromagnetic field in the plasma generated by a moving source of the form (3.8) under conditions (3.11), (3.12), (3.13). The phase $S$ is

$$
S(t, \boldsymbol{x}, \omega, \tau)=\frac{\sqrt{\omega^{2}-\omega_{p}^{2}}}{c_{0}}\left|\boldsymbol{x}-\boldsymbol{x}_{0}(\tau)\right|-\omega(t-\tau)-\omega_{0} \tau .
$$

We suppose that $\omega_{0}>\omega_{p}$. System (3.23) accepts the form

$$
\frac{\left|\boldsymbol{x}-\boldsymbol{x}_{0}(\tau)\right|}{c_{0} \sqrt{1-\frac{\omega_{p}^{2}}{\omega^{2}}}}-(t-\tau)=0, \quad-\frac{\sqrt{\omega^{2}-\omega_{p}^{2}}}{c_{0}} v(\boldsymbol{x}, \tau)+\left(\omega-\omega_{0}\right)=0,
$$

and under the condition

$$
\sup _{t, \omega \geq \omega_{p}} \frac{|\boldsymbol{v}(t)|}{v_{g}(\omega)}<1
$$

system (4.4) has an unique solution $\left(\omega_{s}, \tau_{s}\right)$ such that $\omega_{s}>\omega_{p}$.

The substitution $\left(\omega_{s}, \tau_{s}\right)$ in formulae (3.21), (3.22) gives the expression for the electromagnetic field generated by the moving source.

Example 2. Let $\boldsymbol{v}$ be a constant vector and let $v(\boldsymbol{x}, t)= \pm|\boldsymbol{v}|$. In this case equations (4.4) accept the form

$$
\tau=t-\frac{\left|\boldsymbol{x}-\boldsymbol{x}_{0}(\tau)\right|}{c_{0} \sqrt{1-\frac{\omega_{p}^{2}}{\omega^{2}}}}, \quad \omega=\omega_{0} \pm \frac{\sqrt{\omega^{2}-\omega_{p}^{2}}}{c_{0}}|\boldsymbol{v}|,
$$

where the sign + is taken if the source moving to the receiver and the sign - if the source moving from the receiver. We obtain from second equation in (4.5) that

$$
\omega_{s}^{ \pm}=\frac{1}{1-M^{2}}\left(\omega_{0} \pm M \sqrt{\omega_{0}^{2}-\left(1-M^{2}\right) \omega_{p}^{2}}\right)
$$

where $M=\frac{|\boldsymbol{v}|}{c_{0}}(<1)$ is the Mach number. For $\omega=\omega_{s}^{ \pm}$first equation in (4.5) has the unique solution $\tau_{s}^{ \pm}$. It is easy to see that

$$
\operatorname{det} S^{\prime \prime}\left(t, \boldsymbol{x}, \omega_{s}^{ \pm}, \tau_{s}^{ \pm}\right)=-\left(1 \pm \frac{|\boldsymbol{v}|}{c_{0} \sqrt{1-\frac{\omega_{p}^{2}}{\left(\omega_{s}^{ \pm}\right)^{2}}}}\right)^{2}
$$

and

$$
\operatorname{sgn} S^{\prime \prime}\left(t, \boldsymbol{x}, \omega_{s}^{ \pm}, \tau_{s}^{ \pm}\right)=0
$$


The substitution $\left(\omega_{s}, \tau_{s}\right)$ and (4.6), (4.7) in formulae (3.21), (3.22) gives the expressions for the electromagnetic field

$$
\begin{aligned}
& \boldsymbol{E}_{ \pm}(t, \boldsymbol{x}) \sim \frac{1}{4 \pi i} \frac{a\left(\tau_{s}^{ \pm}\right) \omega_{s}^{ \pm} \mu}{\left(1 \pm \frac{|\boldsymbol{v}|}{c_{0} \sqrt{1-\frac{\omega_{p}^{2}}{\left(\omega_{s}^{ \pm}\right)^{2}}}}\right)}\left(I+\frac{c_{0}^{2}}{\left(\omega_{s}^{ \pm}\right)^{2}-\omega_{p}^{2}} \nabla_{\boldsymbol{x}} \nabla_{\boldsymbol{x}} \cdot\right) \frac{e^{i S\left(t, \boldsymbol{x}, \omega_{s}^{ \pm}, \tau_{s}^{ \pm}\right)}}{\left|\boldsymbol{x}-\boldsymbol{x}_{0}\left(\tau_{s}\right)\right|} \boldsymbol{v} \\
& \boldsymbol{H}_{ \pm}\left(t, \boldsymbol{x} \sim \frac{1}{4 \pi} \nabla_{\boldsymbol{x}} \times\left(\frac{e^{i S\left(t, \boldsymbol{x}, \omega_{s}^{ \pm}, \tau_{j}\right)}}{\left|\boldsymbol{x}-\boldsymbol{x}_{0}\left(\tau_{j}\right)\right|} \boldsymbol{v}\right) \frac{a\left(\tau_{s}^{ \pm}\right)}{\left(1 \pm \frac{|\boldsymbol{v}|}{c_{0} \sqrt{1-\frac{\omega_{p}^{2}}{\left(\omega_{s}^{ \pm}\right)^{2}}}}\right)},\right.
\end{aligned}
$$

for $t=\frac{T}{\lambda},\left|\boldsymbol{x}-\boldsymbol{x}_{0}(t)\right|=\frac{\left|\boldsymbol{X}-\boldsymbol{X}_{0}(T)\right|}{\lambda}, \lambda \rightarrow \infty$.

\subsection{Cherenkov radiation}

Now we apply the above developed approach to consider a field radiation from a moving with a constant velocity $\boldsymbol{v}$ charged particle. Here $e$ is a particle charge, while $c(\omega)>0$ is a field phase velocity in the isotropic dispersive medium. Following [29, Chapter XIV] we use

$$
\rho(t, \boldsymbol{x})=e \delta(\boldsymbol{x}-\boldsymbol{v} t), \quad \boldsymbol{j}(t, \boldsymbol{x})=\boldsymbol{v} e \delta(\boldsymbol{x}-\boldsymbol{v} t) .
$$

We suppose that $\boldsymbol{v}=(0,0, v)$ and $\boldsymbol{x}=\left(x_{1}, x_{2}, x_{3}\right)$. For the particle eigenfrequency case $\omega_{0}=0$ the phase $S$ reads

$$
S(t, \boldsymbol{x}, \omega, \tau)=k(\omega)|\boldsymbol{x}-\boldsymbol{v} t|-\omega(t-\tau) .
$$

Hence system (3.23) accepts the form

$$
\begin{aligned}
& \frac{\partial S(t, \boldsymbol{x}, \omega, \tau)}{\partial \tau}=-\frac{\omega}{c(\omega)} v(\boldsymbol{x}, \tau)+\omega=0, \\
& \frac{\partial S(t, \boldsymbol{x}, \omega, \tau)}{\partial \omega}=\frac{|\boldsymbol{x}-\boldsymbol{v} \tau|}{v_{g}(\omega)}-(t-\tau)=0 .
\end{aligned}
$$

We suppose that the system (4.8), (4.9) has a solution $\left(\omega_{s}, \tau_{s}\right)$ with a non-trivial frequency $\omega_{s}>0$. So, is there exists a pair $\left(\omega_{s}, \tau_{s}\right)$ such that

$$
v\left(\boldsymbol{x}, \tau_{s}\right)=|\boldsymbol{v}| \cos \varphi\left(\boldsymbol{x}, \tau_{s}\right)=c\left(\omega_{s}\right)>0,
$$

where $\varphi(\boldsymbol{x}, \tau)$ is the angle between $\boldsymbol{v}$ and $\boldsymbol{x}-\boldsymbol{v} \tau$. The equation (4.10) can be satisfied if the value of the projection of the velocity $\boldsymbol{v}$ on the vector $\boldsymbol{x}-\boldsymbol{v} t$ is positive.

Let now the pair $\left(\omega_{s}, \tau_{s}\right)$ be an isolated non-degenerated stationary point of the phase $S$,

$$
\begin{aligned}
& v\left(\boldsymbol{x}, \tau_{s}\right)=c\left(\omega_{s}\right), \\
& \tau_{s}=t-\frac{\left|\boldsymbol{x}-\boldsymbol{v} \tau_{s}\right|}{v_{g}\left(\omega_{s}\right)},
\end{aligned}
$$

and $\operatorname{det} S^{\prime \prime}\left(t, \boldsymbol{x}, \omega_{s}, \tau_{s}\right) \neq 0$.

Accordingly to the causality principle the root $\tau_{s}$ of the equation (4.12) has to be positive. It implies the Cherenkov cone condition

$$
v t-x_{3}-\left|\boldsymbol{x}^{\prime}\right| \sqrt{\left|\beta^{2}\left(\omega_{s}\right)-1\right|}>0,
$$


where $\boldsymbol{x}^{\prime}=\left(x_{1}, x_{2}\right)$ and

$$
\beta\left(\omega_{s}\right)=\frac{v}{v_{g}\left(\omega_{s}\right)}
$$

(see, e.g., [1] and references therein). Condition (4.13) connects the time $t$ and position $\boldsymbol{x}=$ $\left(x_{1}, x_{2}, x_{3}\right)$ of the point where the Cherenkov radiation exists.

The substitution of $\left(\omega_{s}, \tau_{s}\right)$ to equations (3.21), (3.22) leads to the following expressions for the electromagnetic fields $\boldsymbol{E}_{s}(t, \boldsymbol{x}), \boldsymbol{H}_{s}(t, \boldsymbol{x})$ (at the point $(t, \boldsymbol{x})$ ) emitted by the moving charge with the instantaneous frequency $\omega_{s}=\omega_{s}(t, \boldsymbol{x})>0$ (the Cherenkov radiation)

$$
\begin{aligned}
\boldsymbol{H}_{s}(t, \boldsymbol{x}) \sim & \frac{1}{4 \pi} \nabla_{\boldsymbol{x}} \times\left(\frac{e^{i S\left(t, \boldsymbol{x}, \omega_{s}, \tau_{s}\right)}}{\left.\mid \boldsymbol{x}-\boldsymbol{v} \tau_{s}\right) \mid} \boldsymbol{v}\right) \frac{e^{\frac{i \pi}{4} \operatorname{sgn} S^{\prime \prime}\left(t, \boldsymbol{x}, \omega_{s}, \tau_{s}\right)}}{\left|\operatorname{det} S^{\prime \prime}\left(t, \boldsymbol{x}, \omega_{s}, \tau_{s}\right)\right|^{1 / 2}} \\
& \times \Theta\left(v t-x_{3}-\left|\boldsymbol{x}^{\prime}\right| \sqrt{\left|\beta^{2}\left(\omega_{s}\right)-1\right|}\right), \\
\boldsymbol{E}_{s}(t, \boldsymbol{x}) \sim & \frac{1}{4 \pi i} \omega_{s} \mu\left(\omega_{s}\right)\left(I+\frac{1}{k^{2}\left(\omega_{s}\right)} \nabla_{\boldsymbol{x}} \nabla_{\boldsymbol{x}} \cdot\right) \frac{e^{i S\left(t, \boldsymbol{x}, \omega_{s}, \tau_{s}\right)}}{\left|\boldsymbol{x}-\boldsymbol{v} \tau_{s}\right|} \boldsymbol{v} \\
& \times \frac{e^{\frac{i \pi}{4} \operatorname{sgn} S^{\prime \prime}\left(t, \boldsymbol{x}, \omega_{s}, \tau_{s}\right)}}{\left|\operatorname{det} S^{\prime \prime}\left(t, \boldsymbol{x}, \omega_{s}, \tau_{s}\right)\right|^{1 / 2}} \Theta\left(v t-x_{3}-\left|\boldsymbol{x}^{\prime}\right| \sqrt{\left|\beta^{2}\left(\omega_{s}\right)-1\right|}\right),
\end{aligned}
$$

where $\Theta(r)$ is the Heaviside function that is

$$
\Theta(r)= \begin{cases}1, & r>0 \\ 0, & r \leq 0\end{cases}
$$

\section{Doppler effect in metamaterials. Numerical example}

Further investigations of the field properties in a dispersive medium already requires the knowledge of the spectral properties of the material refraction index. So, for the further we have to choose the type of material dispersion, in recent literature normally the Lorenz or Drude models, see $[14,31]$ and references therein. Never the less, it is still difficult to investigate the properties of the electromagnetic waves in such dispersive model analytically. (We note that recently some semi-analytic methods were developed [53]). As the example of the developed approach, below we apply the numerics to study the spectral properties of the Doppler effect in a dispersive medium. Here we concentrate on the dispersive metamaterials case where the refraction index $n$ can be negative $\operatorname{Re}(n)<0$ (NIM). (In literature also refer to such a material as being left-handed (LH) material). We consider such a medium characterized by a (relative) permittivity $\varepsilon(\omega)$ and a (relative) permeability $\mu(\omega)$, both of which are complex functions of frequency $\omega$ and the refraction index $n(\omega)$ satisfying the relations $[15,57]$

$$
n(\omega)=\sqrt{|\varepsilon(\omega) \mu(\omega)|} e^{i\left[\phi_{\varepsilon}(\omega)+\phi_{\mu}(\omega)\right] / 2} .
$$

In order to allow a frequency dependence of the refractive index $n$, let us restrict our attention to a single-resonance permittivity

$$
\varepsilon(\omega)=1+\frac{\omega_{\mathrm{P} e}^{2}}{\omega_{\mathrm{T} e}^{2}-\omega^{2}-i \omega \gamma_{e}}
$$

and a single-resonance permeability

$$
\mu(\omega)=1+\frac{\omega_{\mathrm{P} m}^{2}}{\omega_{\mathrm{T} m}^{2}-\omega^{2}-i \omega \gamma_{m}},
$$



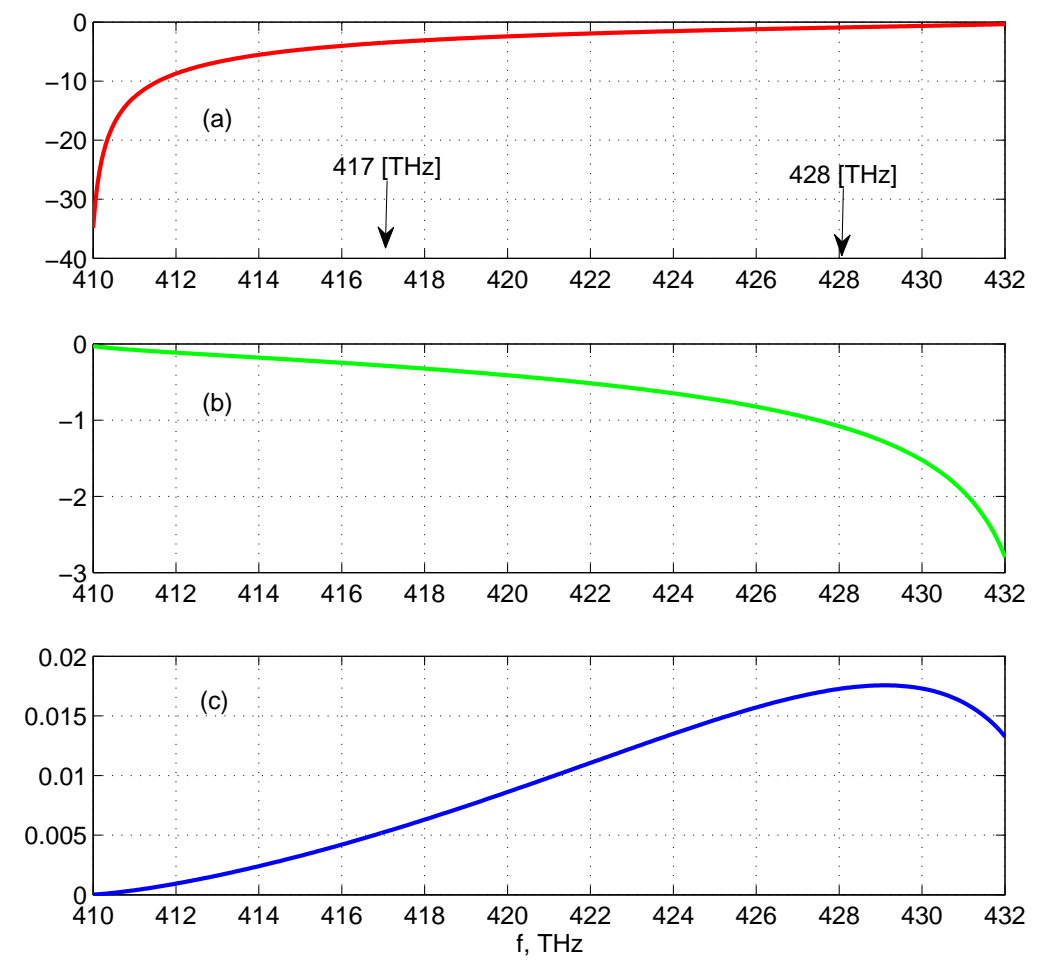

Figure 1. (Color online.) Frequency dependence (a) metaterial refractive index $n(\omega)$ in the frequency interval with $\operatorname{Re} n(\omega)<0$; (b) phase velocity $v_{p}(\omega)$; (c) group velocity $v_{g}(\omega)$, where $\omega=2 \pi f$.

where $\omega_{\mathrm{P} e}, \omega_{\mathrm{P} m}$ are the coupling strengths, $\omega_{\mathrm{T} e}, \omega_{\mathrm{T} m}$ are the transverse resonance frequencies, and $\gamma_{e}, \gamma_{m}$ are the absorption parameters. Both the permittivity and the permeability satisfy the Kramers-Kronig relations [15,57]. The following typical parameters of metamaterial were used for our numerics: $f_{\mathrm{P} e}=298.42 \mathrm{THz}, \gamma_{e}=0.04 \mathrm{THz}, f_{\mathrm{T} e}=409.82 \mathrm{THz}, f_{\mathrm{P} m}=171.09 \mathrm{THz}$, $\gamma_{m}=0.04 \mathrm{THz}, f_{\mathrm{T} m}=397.89 \mathrm{THz}$.

Fig. 1(a) shows the frequency dependence of NIM refractive index $n(\omega)(\omega=2 \pi f)$. Here the permittivity $\varepsilon(\omega)$ and the permeability $\mu(\omega)$ being respectively given by equations (5.2) and (5.3) in the frequency interval from $410 \mathrm{THz}$ to $432 \mathrm{THz}$ where $\operatorname{Re} n(\omega)<0$. It is worth noting that the negative real part of the refractive index is typically observed together with strong dispersion, so that absorption cannot be disregarded in general. However, in a recent experiment [56], it was demonstrated that the incorporation of gain material in a metamaterial makes it possible to fabricate an extremely low-loss and active optical devices. Thus, the original loss-limited negative refractive index can be drastically improved with loss compensation in the visible wavelength range. Following this result we will neglect the imaginary part $\operatorname{Im}(n)$. Fig. 1(b) shows corresponding frequency dependence of the phase velocity $v_{p}(\omega)$ that is negative in considered frequency range, and Fig. 1(c) exhibits the frequency dependence of the group velocity $v_{g}(\omega)$. Further for numerics we renormalized the variables for $\tau$ and $x$ with the scales $l_{0} / c$ and $l_{0}$ respectively, velocities are normalized with the vacuum light velocity $c$, and $l_{0}=75 \mathrm{~nm}$ is the typical spatial scale used at the metamaterial experiments [56]. To seek for simplicity, further we consider with details a case when the position of observer $\boldsymbol{x}$ and the trajectory of a source $\boldsymbol{v}$ are in the same plane $\left(x_{3}=0\right.$ and $\left.H=0\right)$. In this case the geometry becomes $2 \mathrm{D}$ one and the solution to equations (3.23) for $\omega$ reads

$$
\omega=\left(c r^{2} \pm \sqrt{v^{2} r^{2}\left(-x_{2}+v \tau\right)^{2} n(\omega)^{2}}\right) \frac{c \omega_{0}}{c^{2} r^{2}-p_{2}}
$$


where $p_{2}=n(\omega)^{2} v^{2}\left(x_{2}-v \tau\right)^{2}$, and $r=\sqrt{x_{1}^{2}+\left(x_{2}-v \tau\right)^{2}}$. The solution of equations (3.23) for $\tau$ can be written as

$$
\tau=\frac{x_{2} v-v_{g}^{2} t-\sqrt{q_{1}+q_{2}}}{v^{2}-v_{g}^{2}},
$$

where $q_{1}=\left(v^{2}-v_{g}^{2}\right) x_{1}^{2}$, and $q_{2}=v_{g}^{2}\left(x_{2}-v t\right)^{2}$. In simplest $1 \mathrm{D}$ situation with $x_{1}=0$ from (5.4), (5.5) we have two equations

$$
\omega=\frac{\omega_{0}}{1 \pm n(\omega) v}, \quad \tau=\frac{x_{2}-v_{g} t}{v-v_{g}} .
$$

First formula in equation (5.6) describes the well-known Doppler effect, when the shift of $\omega$ depends on the $n v$ signum. For conventional materials with $n>0$ (in this case we have to choose upper + sign) the received frequency $\omega$ is lower (compared to the emitted frequency $\omega_{0}$ ) during the source approach $v>0$, and $\omega$ is higher during the source recession $v<0$. However the situation becomes more complicated in dispersive metamaterials with negative refraction index (NIM) where $\operatorname{Re}(n(\omega))<0$ with the refraction index depending on the frequency $n=n(\omega)$ were both phase and group velocities are the frequency functions. In dispersive medium the first relation in (5.4) and (5.5) become coupled equations. Even in a simple 1D geometry the first equation for frequency $\omega$ in (5.6) already requires the spectral details of the medium refraction index $n(\omega)$. Moreover, in the second equation in (5.6) the group velocity $v_{g}$ becomes the frequency function, thus, the retardation time $\tau$ will be different for different source eigenfrequency $\omega_{0}$.

In our numerical simulations we search for the solution of equations (5.1)-(5.6) for $\omega$ and $\tau$ at the fixed position $x_{1}, x_{2}$ and the time $t$ of an observer. First we studied more simple 1D dispersive NIM, equation (5.6). This equation is solved by the standard numerical methods [38, 49], and resulting dependencies ares shown in Fig. 2. Fig. 2(a) shows the shifted frequency $\omega(\omega=2 \pi f)$ vs the source frequency $\omega_{0}$ for area where $n(\omega)<0$. Further such a calculated dependence $\omega=\omega\left(\omega_{0}\right)$ allows us to evaluate the group velocity $v_{g}(\omega)$ and, finely the retardation time $\tau=\tau\left(\omega_{0}\right)$ in Fig. 2(b). We observe from Fig. 2(a), (b) that both dependencies $\omega=\omega\left(\omega_{0}\right)$ and $\tau=\tau\left(\omega_{0}\right)$ have pronounced nonlinear shape that certainly is determined by a dispersive spectrum of used metamaterial. Let us remind that in dispersiveless case both Dopple's frequency shift and the retardation time depend on the particle velocity $v$ only.

The 2D geometry is more complicated. In this situation we have to solve numerically the equations (5.4), (5.5) (with added material relations (5.1)-(5.3)) that becomes a strong nonlinear system. It is worth to note that in this case the Newton's method for solving nonlinear equations has an unfortunate tendency to wander off $[38,49]$ if the initial guess is not sufficiently close to the root. In order to evaluate the solution of such a system the globally convergent multidimensional Newton's method was applied [38,49]. The radiated source has $v=0.5$, and $f_{0}=420 \mathrm{THz}$. The observer point is at $x_{1}=0.01, x_{2}=1.595$, and the time is $t=2$. The result of calculations is $f=417.82 \mathrm{THz}$, and the $\tau=3.1901$. This point is indicated in Fig. 1(a) with $f=417.82 \mathrm{THz}$, and corresponds to $v_{p}=-0.31673, v_{g}=0.0084029$, and $x_{2}<v \tau$. Other solution was obtained for parameters $x_{1}=0, x_{2}=1.595$ that results $f=428.9 \mathrm{THz}$ and $\tau=3.1694$ (see arrows in Fig. 1(a)).

\section{Conclusion}

In this paper the time-frequency integrals and the two-dimensional stationary phase method are applied to study the electromagnetic waves radiated by moving modulated sources in dispersive media. We show that such unified approach leads to explicit expressions for the field amplitudes and simple relations for the field eigenfrequencies and the retardation time that become the 

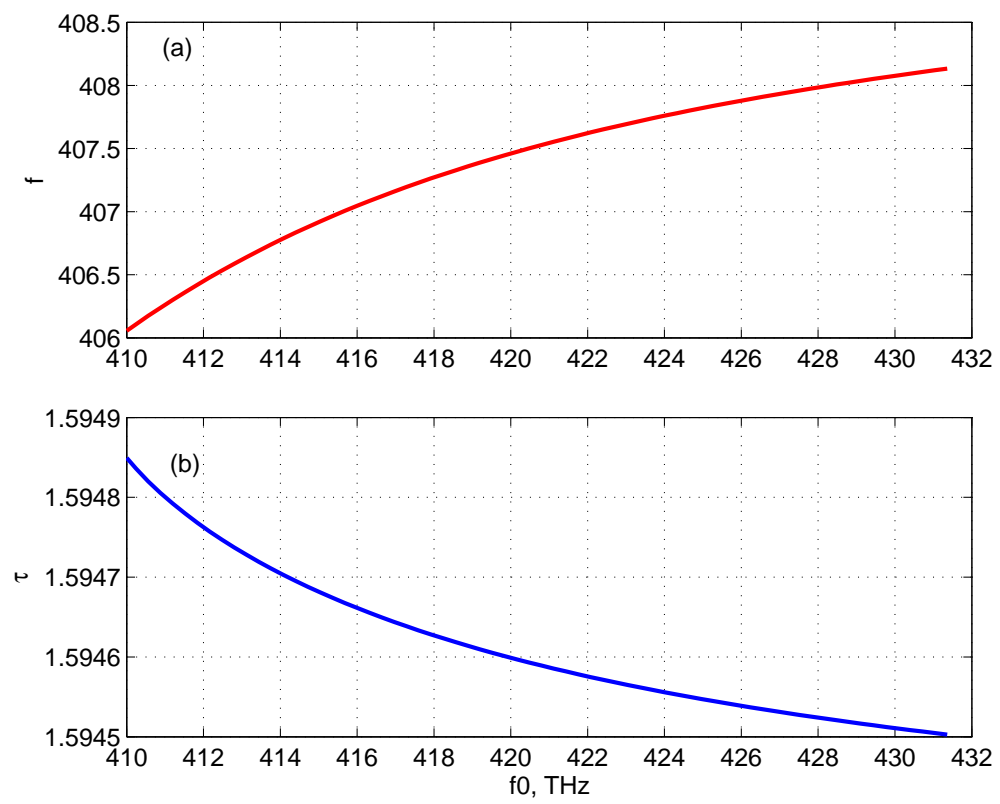

Figure 2. (Color online.) Dependence of the Doppler shifted frequency $\omega(\omega=2 \pi f)$ (a), and the time $\tau(\mathrm{b})$ on the source frequency $\omega_{0}$ for area where $n(\omega)<0$. See details in text.

coupled variables. The main features of the technique are illustrated by examples of the moving source fields in the plasma and the Cherenkov radiation. In the paper it is emphasized that deeper comprehension and insight the wave effects in dispersive case already requires the explicit formulation of the dispersive material model. As the advanced application we have considered the Doppler frequency shift in a complex single-resonant dispersive metamaterial (Lorenz) model where in some frequency ranges the negativity of the real part of the refraction index can be reached. We have demonstrated that in a dispersive case the Doppler frequency shift acquires a nonlinear dependence on the modulating frequency of the radiated particle. The detailed frequency dependence of such a shift and spectral behavior of phase and group velocities (that have the opposite directions) are studied numerically. Such dependence in principle can be used also for reconstruction of unknown parameters of the dispersive medium. Other challenge is to enforce the developed approach to the Cerenkov radiation and its peculiar features in the dispersive and left-handed media. Such a problem will be considered in future study.

\section{Acknowledgements}

The work of authors is partially supported by PROMEP, grant Redes CA 2011-2012. The work of G.B. is partially supported by CONACyT grant 169496. The work of V.R. was partially supported by CONACyT grant 179872 .

\section{References}

[1] Afanasiev G.N., Vavilov-Cherenkov and synchrotron radiation. Foundations and applications, Fundamental Theories of Physics, Vol. 142, Kluwer Academic Publishers, New York, 2005.

[2] Afanasiev G.N., Kartavenko V.G., Radiation of a point charge uniformly moving in a dielectric medium, J. Phys. D: Appl. Phys. 31 (1998), 2760-2776.

[3] Afanasiev G.N., Kartavenko V.G., Magar E.N., Vavilov-Cherenkov radiation in dispersive medium, Phys. B 269 (1999), 95-113.

[4] Babich V.M., Buldyrev V.S., Molotkov I.A., The space-time ray method, Leningrad. Univ., Leningrad, 1985. 
[5] Belonogaya E.S., Galyamin S.N., Tyukhtin A.V., Properties of Vavilov-Cherenkov radiation in an anisotropic medium with a resonant dispersion of permittivity, J. Opt. Soc. Amer. B. 28 (2011), 2871-2878.

[6] Blestein N., Handelsman R.A., Asymptotic extention of integrals, Dover Publication, New York, 1975.

[7] Bolotovskii B.M., Vavilov-Cherenkov radiation: its discovery and applications, Phys. Usp. 52 (2009), 10991110.

[8] Brillouin L., Wave propagation and group velocity, Pure and Applied Physics, Vol. 8, Academic Press, New York, 1960.

[9] Carusotto I., Artoni M., La Rocca G.C., Bassani F., Slow group velocity and Cherenkov radiation, Phys. Rev. Lett. 87 (2001), 064801, 4 pages.

[10] Chen J., Wang Y., Jia B., Geng T., Li X., Feng L., Qian W., Liang B., Zhang X., Gu M., Zhuang S., Observation of the inverse Doppler effect in negative-index materials at optical frequencies, Nature Photonics 5 (2011), 239-242.

[11] Choi M., Lee, Seung H., Kim Y., Kang Seung B., Shin J., Kwak M.H., Kang K.Y., Lee Y.H., Park N., Min B., A terahertz metamaterial with unnaturally high refractive index, Nature 470 (2011), 369-373.

[12] Cohen L., Time-frequency analysis, Prentice-Hall Inc., Englewood Cliffs, N.J., 1995.

[13] Doil'nitsina E.G., Tyukhtin A.V., Radiated power of oscillators traveling in a moving medium, Radiophys. Quantum Electron. 50 (2007), 287-298.

[14] Duan Z., Wu B.I., Xi S., Chen H., Chen M., Research progress in reversed Cherenkov radiation in doublenegative metamaterials, Progr. Electromag. Res. 90 (2009), 75-87.

[15] Dung H.T., Buhmann S.Y., Knöll L., Welsch D.G., Scheel S., Kästel J., Electromagnetic-field quantization and spontaneous decay in left-handed media, Phys. Rev. A 68 (2003), 066606, 15 pages, quant-ph/0306028.

[16] Fedoryuk M.V., Method of the steepest descent, Nauka, Moscow, 1977.

[17] Felsen L.B., Marcuvitz N., Radiation and scattering of waves, Prentice-Hall Microwaves and Fields Series, Prentice-Hall Inc., Englewood Cliffs, N.J., 1973.

[18] Galyamin S.N., Tyukhtin A.V., Electromagnetic field of a moving charge in the presence of a left-handed medium, Phys. Rev. B 81 (2010), 235134, 14 pages.

[19] Ginzburg V.L., Propagation of electromagnetic waves in plasma, Nauka, Moscow, 1960.

[20] Ginzburg V.L., Radiation by uniformly moving sources (Vavilov-Cherenkov effect, transition radiation, and other phenomena), Phys. Usp. 39 (1996), 973-982.

[21] Ginzburg V.L., Theoretical physics and astrophysics, 2nd ed., Nauka, Moscow, 1981.

[22] Grudsky S.M., Obrezanova O.A., Rabinovich V.S., Sound propagation from a moving air source in the ocean covered by ice, Acoustical Phys. 41 (1995), 1-6.

[23] Il'ichev V.I., Rabinovich V.S., Rivelis E.A., Hoha U.V., Acoustic field of a moving narrow-band source in oceanic waveguides, Dokl. Phys. 304 (1989), 1123-1127.

[24] Jackson J.D., Classical electrodynamics, 2nd ed., John Wiley \& Sons Inc., New York, 1975.

[25] Jelley J.V., Cherenkov radiation and its applications, Pergamon Press, London, 1958.

[26] Landau L.D., Lifshits E.M., Course of theoretical physics, Vol. 2, The classical theory of fields, 4th ed., Pergamon Press, Oxford, 1975.

[27] Landau L.D., Lifshits E.M., Course of theoretical physics, Vol. 9, Statistical physics, Part 2, Theory of the condensed state, Pergamon Press, Oxford, 1981.

[28] Landau L.D., Lifshits E.M., Theoretical physics, Vol. 2, Field theory, Nauka, Moscow, 1988.

[29] Landau L.D., Lifshits E.M., Theoretical physics, Vol. 8, Electrodynamics of continuous media, Nauka, Moscow, 1982.

[30] Lewis R.M., Asymptotic methods for the solution of dispersive hyperbolic equations, in Asymptotic Solutions of Differential Equations and Their Applications, Wiley, New York, 1964, 53-107.

[31] Lu J., Grzegorczyk T., Zhang Y., Pacheco J., Wu B.I., Kong J., Chen M., Cherenkov radiation in materials with negative permittivity and permeability, Opt. Express 11 (2003), 723-734.

[32] Luo C., Ibanescu M., Johnson S.G., Joannopoulos J.D., Cherenkov radiation in photonic crystals, Science 299 (2003), 368-371.

[33] Melamed T., Felsen L.B., Pulsed-beam propagation in lossless dispersive media. I. Theory, J. Opt. Soc. Amer. A 15 (1993), 1268-1276. 
[34] Obrezanova O.A., Rabinovich V.S., Acoustic field generated by moving sources in stratified waveguides, Wave Motion 27 (1998), 155-167.

[35] Obrezanova O.A., Rabinovich V.S., Acoustic field of a source moving along stratified waveguide surface, Acoustical Phys. 39 (1993), 517-521.

[36] Pendry J.B., Holden A.J., Robbins D.J., Stewart W.J., Magnetism from conductors and enhanced nonlinear phenomena, IEEE Trans. Microwave Theory Tech. 47 (1999), 2075-2084.

[37] Pendry J.B., Schurig D., Smith D.R., Controlling electromagnetic fields, Science 312 (2006), $1780-1782$.

[38] Press W.H., Teukolsky S.A., Vetterling W.T., Flannery B.P., Numerical recipes. The art of scientific computing, 3rd ed., Cambridge University Press, Cambridge, 2007.

[39] Shalaev V.M., Optical negative-index metamaterials, Nature Photonics 1 (2007), 41-48, arXiv:1109.0084.

[40] Shin J., Shen J.T., Fan S., Three-dimensional metamaterials with an ultrahigh effective refractive index over a broad bandwidth, Phys. Rev. Lett. 102 (2009), 093903, 4 pages.

[41] Shubin M.A., Pseudodifferential operators and spectral theory, 2nd ed., Springer-Verlag, Berlin, 2001.

[42] Smith D.R., Padilla J.W., Vier D.C., Nemat-Nasser S.C., Schultz S., Composite medium with simultaneously negative permeability and permittivity, Phys. Rev. Lett. 84 (2000), 4184-4187.

[43] Smolyaninov I.I., Hung Y.J., Davis C.C., Magnifying superlens in the visible frequency range, Science 315 (2007), 1699-1701, physics/0610230.

[44] Sommerfeld A., Mechanics of deformable bodies. Lectures on theoretical physics, Vol. II, Academic Press Inc., New York, 1950.

[45] Soukoulis C.M., Wegener M., Past achievements and future challenges in the development of threedimensional photonic metamaterials, Nature Photonics 5 (2011), 523-530, arXiv:1109.0084.

[46] Stevens T.E., Wahlstrand J.K., Kuhl J., Merlin R., Cherenkov radiation at speeds below the light threshold: phonon-assisted phase matching, Science 291 (2001), 627-630.

[47] Stix T.H., Waves in plasmas, Springer-Verlag, New York, 1992.

[48] Swanson D.G., Plasma waves, 2nd ed., IOP Publishing, Bristol, 2003.

[49] Taflove A., Hagness S.C., Computational electrodynamics: the finite-difference time-domain method, 2nd ed., Artech House Inc., Boston, MA, 2000.

[50] Tyukhtin A.V., Energy characteristics of radiation from an oscillating dipole moving in a dielectric medium with resonant dispersion, Tech. Phys. 50 (2005), 1084-1088.

[51] Tyukhtin A.V., Galyamin S.N., Vavilov-Cherenkov radiation in passive and active media with complex resonant dispersion, Phys. Rev. E 77 (2008), 066606, 8 pages.

[52] Veselago V.G., The electrodynamics of substances with simultaneously negative values of $\epsilon$ and $\mu$, Sov. Phys. Usp. 10 (1968), 509-514.

[53] Vorobev V.V., Tyukhtin A.V., Cherenkov radiation in a wire metamaterial, Phys. Rev. Lett. 108 (2012), 184801, 4 pages.

[54] Waters K.R., Hughes M.S., Brandenburger G.H., Miller J.G., On a time-domain representation of the Kramers-Krönig dispersion relations, J. Acoust. Soc. Amer. 108 (2000), 2114-2119.

[55] Xi S., Chen H., Jiang T., Ran L., Huangfu J., Wu B.I., Kong J.A., Chen M., Experimental verification of reversed Cherenkov radiation in left-handed metamaterial, Phys. Rev. Lett. 103 (2009), 093903, 4 pages.

[56] Xiao S., Drachev V.P., Kildishev A.V., Ni X., Chettiar U.K., Hsiao-Kuan Y., Shalaev V.M., Loss-free and active optical negative-index metamaterials, Nature 466 (2010), 735-738.

[57] Ziolkowski R.W., Heyman E., Wave propagation in media having negative permittivity and permeability, Phys. Rev. E 64 (2001), 056625, 15 pages. 